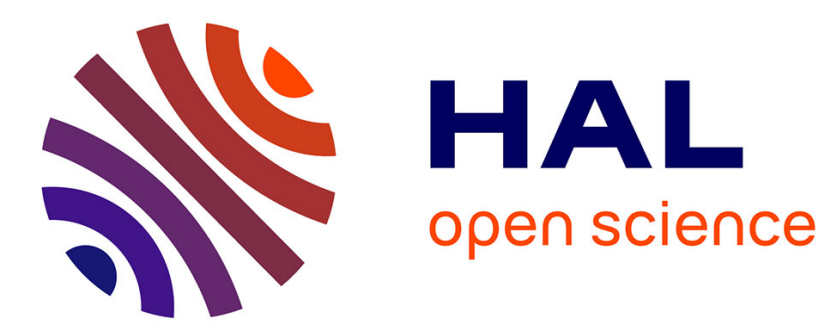

\title{
INTERACTIONS OF INTENSE MICROWAVES WITH RYDBERG ATOMS
}

\author{
P. Koch
}

\section{To cite this version:}

P. Koch. INTERACTIONS OF INTENSE MICROWAVES WITH RYDBERG ATOMS. Journal de Physique Colloques, 1982, 43 (C2), pp.C2-187-C2-210. 10.1051/jphyscol:1982215 . jpa-00221826

\section{HAL Id: jpa-00221826 https://hal.science/jpa-00221826}

Submitted on 1 Jan 1982

HAL is a multi-disciplinary open access archive for the deposit and dissemination of scientific research documents, whether they are published or not. The documents may come from teaching and research institutions in France or abroad, or from public or private research centers.
L'archive ouverte pluridisciplinaire HAL, est destinée au dépôt et à la diffusion de documents scientifiques de niveau recherche, publiés ou non, émanant des établissements d'enseignement et de recherche français ou étrangers, des laboratoires publics ou privés. 


\title{
INTERACTIONS OF INTENSE MICROWAVES WITH RYDBERG ATOMS
}

\author{
P.M. Koch* \\ Physics Department, Yale University, New Haven, CT 06511, U.S.A.
}

\begin{abstract}
Résumé.- Nous présentons une revue d'expériences qui s'intéressent à l'ionisation, l'excitation, et la structure quantique des atomes d'hydrogène et d'hélium très excités plongês dans un champ de micro-onde intense, ainsi qu'une comparaison avec les théories actuelles. Celles-ci sont capables d'expliquer qualitativement certaines structures étudiées, mais elles ne permettent pas encore d'expliquer quantitativement les mesures d'ionisation effectuées à 1 'aide d'un très grand nombre (plusieurs centaines) de photons micro-onde. Il existe des calculs par la méthode de Monte-Carlo basés sur une théorie classique des interactions qui donnent un accord raisonnable avec les expériences d'ionisation publiées. Ces calculs négligent $1^{\prime}$ effet tunnel (quantique) et des expériences sont actuellement en cours pour montrer si cet effet, nëgligê dans 1 'approche classique, joue un rôle important. D'autres expériences récentes montrent que la dépendance de 1 'ionisation en fonction de la puissance microonde d'un atome de Rydberg d'hélium dans l'état triplet $m=0$ est très diffërente de celle d'un atome hydrogène. On est capable actueliement d'expliquer le seuil d'ionisation de l'hêlium en termes d'interactions dynamiques aux croisements évitês successifs du diagramme de potentiel Stark de 1'hêlium.
\end{abstract}

\begin{abstract}
Experiments investigating ionization, excitation, and quanta1 structure of highly-excited hydrogen and helium atoms in an intense microwave field are reviewed in terms of existing theory. Quantal theory is able to explain some of the results of the structure studies but is not yet at the level of producing quantitative agreement with ionization experiments, which involve very large numbers (hundreds) of microwave photons. Published MonteCarlo calculations based on a classical theory of the interactions are in reasonable agreement with the results of published ionization experiments, but additional experiments are being carried out to see if quantal tunneling, which is ignored by the classical theories, plays an important role. New experiments show that the pover dependence of microwave ionization of $m=0$ triplet helium Rydberg atoms is very different from that of hydrogen atoms. We are able to explain the threshold region of ionization in helium in terms of dynamic interactions at the first (and subsequent) avoided crossings(s) of adiabatic helium Stark potential curves.
\end{abstract}

1. Introduction. - This paper reviews briefly our present experimental and theoretical understanding about what happens when an atom with one of its electrons in a highly-excited level with principal quantum number $n \gg>1$ (to be called a Rydberg atom) interacts with an intense microwave electromagnetic field. We shall see that much remains to be understood about this problem, both qualitatively and quantitatively. We shall confine the discussion primarily to the one-electron hydrogen atom, the subject of nearly all the still rather meager amount of theory that has been done, and to a lesser extent, to the two-electron helium atom, the simplest multi-electron atom. We shall see that hydrogen and helium atoms prepared in Rydberg states with very nearly the same energy are ionized rather differently by the microwave field and shall explain, at least partially, why this is so.

Other review articles by the present author ${ }^{1,2}$ and by Bayfield ${ }^{3-5}$ have covered some of the material presented here. The latter articles ${ }^{4-5}$, especially, reviewed *After 1 sept. 1982 : Physics Department, State University of New York, Stony Brook,
NY 11794 U.S.A. 
a large body of the quantal and semi-quantal theories treating the interactions of atoms and molecules with intense electromagnetic fields. This material will not be reviewed in detail here; the interested reader should consult these earlier reviews.

A few, loose definitions are in order at the outset. We shall take "intense" to mean that the Rydberg atom undergoes multiphoton absorption and emission in the microwave field that can be of rather high order, as much as several hundred. This usually means that induced transitions to continuum states, multiphoton ionization, can complete successfully with those to lower- or higher-lying bound states, multiphoton (de-)excitation; spontaneous transitions generally play a negligible role. Viewed semi-classically, the oscillatory microwave electric field $F_{0} c o s w t$ has a peak strength $F_{0}$ comparable to the mean Coulomb field binding the Rydberg electron to its ionic core.

The full problem may be sub-divided into the questions of (1) atomic "structure", or what are the quantized energy levels of the atom in the microwave field and (2) atomic "transitions", or what are the detailed electronic motions (in a classical sense) among the various atomic states, particularly to the continuum? We shall motivate the conclusion that, to data, quantal or semi-quantal theories have dealt better with various aspects of the structure question but less well quantitatively with the question of ionization (bound-free transitions) than have classical theories, which obviously have nothing to say about (quantal) structure but seem capable of dealing well with some aspects of the non-linear dynamics. Here there are exciting unanswered questions concerning possible "stochastic" ionization mechanisms ${ }^{6-11}$. Of course, any purely classical theory ignores quantal tunneling processes, which play an important role in ionization of atoms by a static electric field. The importance of tunneling in ionization of atoms by oscillatory fields, however, is not yet well understood. Neither is the onset of stochasticity in quantal non-linear dynamical systems.

2. A "Reasonable Approximate Hamiltonian for Hydrogen. - Consider a hydrogen atom with an infinitely massive nucleus. For highly-excited levels $H$ (high $n$ ) with $n>1$, the binding energy of the Bohr levels scales as $\mathrm{n}^{-2}$, whereas the spacing between neighboring n-manifolds and the fine-structure and hyperfine-structure within a given $n$-manifold each scale as $n^{-3}$, albeit with diminishing constants of proportionality. Quantum-electrodynamic splittings (Lamb shifts, etc.) decrease with $n$ even more rapidly. In the rest of this paper, we ignore fine-, hyperfine-, and QLDcontributions to the energies of hydrogen levels. They are much smaller than the Stark interaction energy associated with the oscillatory electric field in an intense microwave field. (In helium, however, we shall include, via a quantum defect formulation, the electrostatic fine-structure because it greatly modifies the atomic Stark structure, particularly for levels with low values of the magnetic quantum number, $|\mathrm{m}|=0,1$. The $|\mathrm{m}|=0$ Stark structure is especially non-hydrogenic. For the most part, the magnetic fine-structure can still be ignored.)

At a given point in free space an applied microwave field consists of an oscillatory electric field $\vec{F}(t)=\vec{F}_{0} \cos \omega t$ and an oscillatory magnetic field $\vec{B}(t)=$ $\overrightarrow{\mathrm{B}}_{\mathrm{o}} \cos \omega \mathrm{t}$. For $\mathrm{H}$ (high $\mathrm{n}$ ) atoms, it is not hard to show that the Zeeman interaction energies with $\vec{B}(t)$ are generally much smaller than the Stark interaction energies with $\vec{F}(t)$. For a plane-wave microwave field, one can show that the ratio of the maximum Zeeman energy $W_{m}$ associated with the electronic orbital magnetic moment and the maximum Stark energy $W_{e}$ is of order

$$
\mathrm{w}_{\mathrm{m}} / \mathrm{w}_{\mathrm{e}} \approx \alpha / 2 \mathrm{n} \text {, }
$$

where $\alpha \simeq(137)^{-1}$ is the fine structure constant. For $n \sim 27$, this ratio is $\sim 10^{-4}$. The Zeeman energy associated with the electronic spin-angular magnetic moment is even smaller.

If the microwave field is contained in a waveguide or cavity, its electric and magnetic components will have spatial dependences which depend on the particular mode being excited. There will exist regions where one field is much larger than the other. The experiments discussed in this paper have been performed only in waveguides or cavities in which the interaction of the atoms with $\overrightarrow{\mathrm{F}}(t)$ was totally dominant over that with $\vec{B}(t)$. 
Thus it is reasonable to adopt as a Hamiltonian (in au)

$$
K(\vec{r}, t)=\vec{p}^{2} / 2-r^{-1}+2 F_{0} \cos \omega t,
$$

which includes only the non-relativistic kinetic energy of the electron and its electric interactions with the point nucleus and with $\vec{F}(t)$. This has been the starting point for all the non-relativistic theories treating hydrogen.

3. Static Electric Field Ionization. - As a prelude to describing the intense microwave experiments, it is useful to review briefly the results of our experiments at Yale on static electric field ionization of $\mathrm{H}(\mathrm{high} \mathrm{n}$ ) atoms in resolved substates described by parabolic quantum numbers $\left(n, n_{1}, n_{2},|m|\right)$. Both kinds of experiments used similar fast-beam methods; in fact, micorwave ionization in a TM020-mode cavity was used as a "black-box," $100 \%$ efficient detector of $\mathrm{H}(\mathrm{high}-\mathrm{n})$ atom in the static ionization experiments.

SCHEMATIC OF APPARATUS

(TOP VIEW)

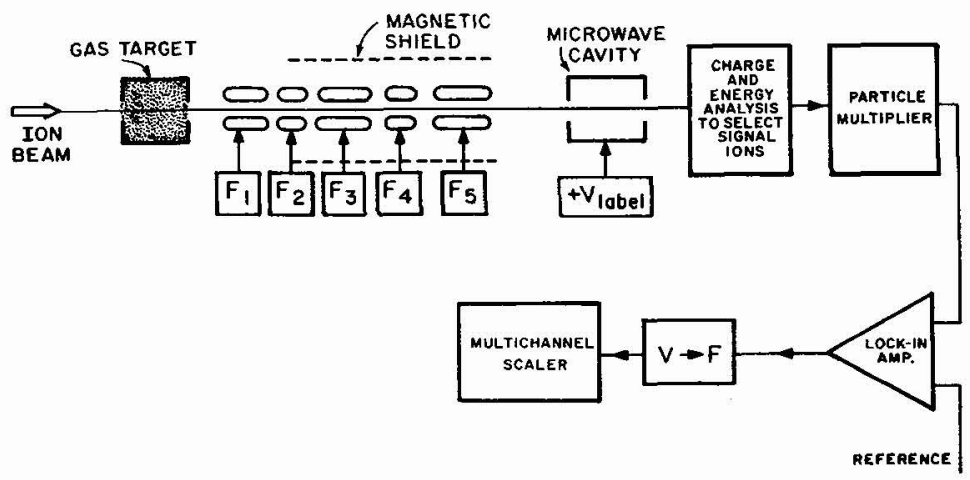

Fig. 1a- A schematic diagram (top view) of the apparatus used for static and microwave ionization experiments with fast beams of hydrogen or helium atoms produced by ion-atom electron-transfer collisions. The various pairs of electrodes labelled $\mathrm{F}_{1}-\mathrm{F}_{5}$ produce static electric fields through which the atoms fly. Two continuous $\mathrm{CO}_{2}$ laser beams may be directed either collinearly with the atomic beam or to cross it in $F_{1}$ and $F_{3}$, respectively, where they drive transitions between Stark substates of different $n$-manifolds. A microwave cavity (or waveguide, not shown) is used to study microwave multiphoton ionization and excitation processes. Experimental analog signals are digitized and signal-averaged in a multichannel scaler.

Fig. la,b shows a schematic diagram of our apparatus. An $\mathrm{H}^{+}$or $\mathrm{He}^{+}$ion beam with laboratory kinetic energy $T \cong 3-20 \mathrm{keV}$ undergoes electron-transfer collisions with target gas atoms, producing Rydberg atoms with approximately an $\mathrm{n}^{-3}$ distribution and an unknown distribution of substates. With use of various combinations of static electric fields $\mathrm{F}_{1}-\mathrm{F}_{5}$, continuous laser beams (argon ion or $\mathrm{CO}_{2}$ ), and techniques reviewed in Ref. 2, a beam of atoms could be prepared in an n-band (a narrowed distribution of $n$-manifolds between $n_{a}$ and $n_{b}$ ), in an individual $n$-manifold with unresolved substates, or in an individual (Stark) substate of a given n-manifold. Fig. 1b shows the most recent, inclined laser beam-particle beam geometry we have been using for double-resonance $\mathrm{CO}_{2}$ laser production of $\mathrm{H}$ (high $\mathrm{n}$ ) and He(high $\mathrm{n}$ ) atoms. Unlike our previous collinear-beam geometry ${ }^{2}, 12$ the inclined geometry allows us to have each laser beam interact with the atoms in only one section of the beam apparatus. Fig. Ia shows a microwave cavity being used to ionize the Rydberg atoms for subsequent detection of the resultant fast ions. We also use ionization in a static axial electric field for detection (not shown in Fig. 1). 


\section{SCHEMATIC OF APPARATUS \\ (SIDE VIEW)}

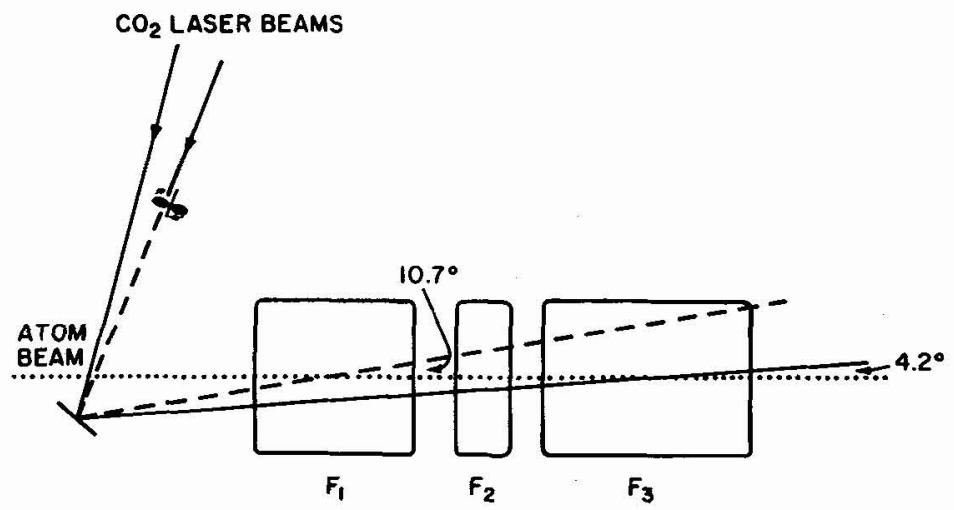

Fig. 1b - A side view of a part of the apparatus shown in Fig. la. It shows the geometry of the two continuous $\mathrm{C}_{2}$ laser beams which cross the fast atomic beam in electric field regions $F_{1}$ and $F_{3}$, respectively.

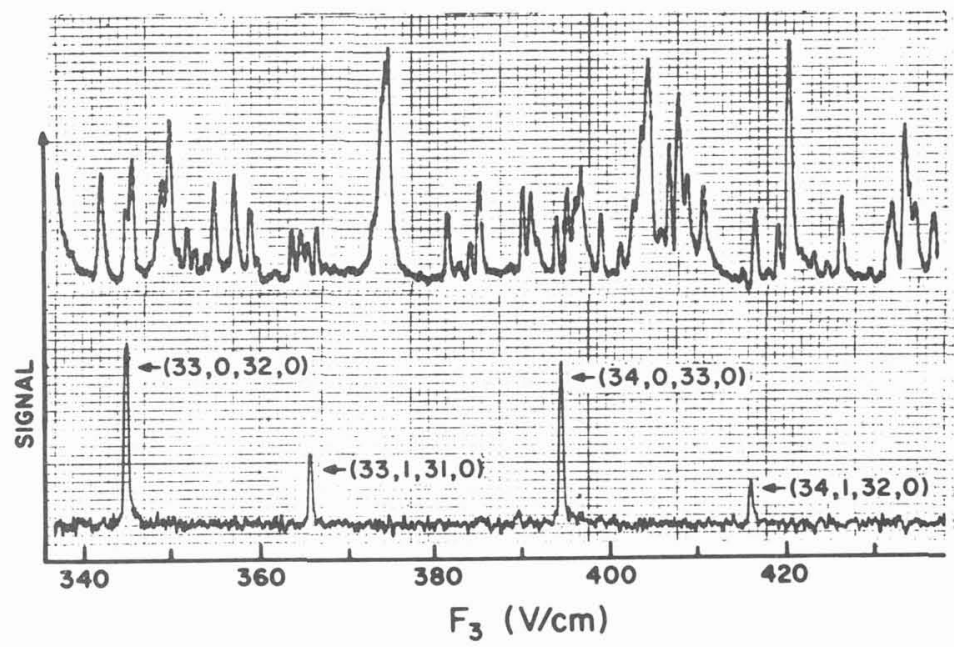

Fig. 2 - Upper trace: A "single-resonance" Stark-tuned spectrum displaying $\mathrm{CO}_{2}$ laser-driven transitions in $F_{1}$ originating on a large number of $H(n=10)$ substates. Lower trace: A "double-resonance" spectrum displaying only those transitions out of the $H(n=10)$ Stark substate $(10,0,9,0)$.

Fig. 2 shows two Stark-tuned spectra ${ }^{2}$ obtained with one collinear $\mathrm{CO}_{2}$ 1aser beam driving transitions between substates of two different pairs of n-manifolds: $n=7$ and $n=10$ in $F_{1}$ and $n=10$ and $n=33,34$ in $F_{3}$. Each $n$-manifold has $n(n+1) / 2$ Stark substates, The "single-resonance" spectrum (detected with one lockin amplifier) registered the many $n=10 \rightarrow n=33,34$ transitions in $F_{3}$ that were connected by appreciable matrix elements, The "double-resonance" spectrum (detected simultaneously with a second lockin amplifier) registered only those driven in $F_{3}$ out of the single $H(n=10)$ substate $(10,0,9,0)$. It was previously "tagged" by the laser- 
driven transition $(7,0,6,0) \rightarrow(10,0,9,0)$ in $F_{1}$. Obvious1y, the double-resonance method allows isolation of individual substates.

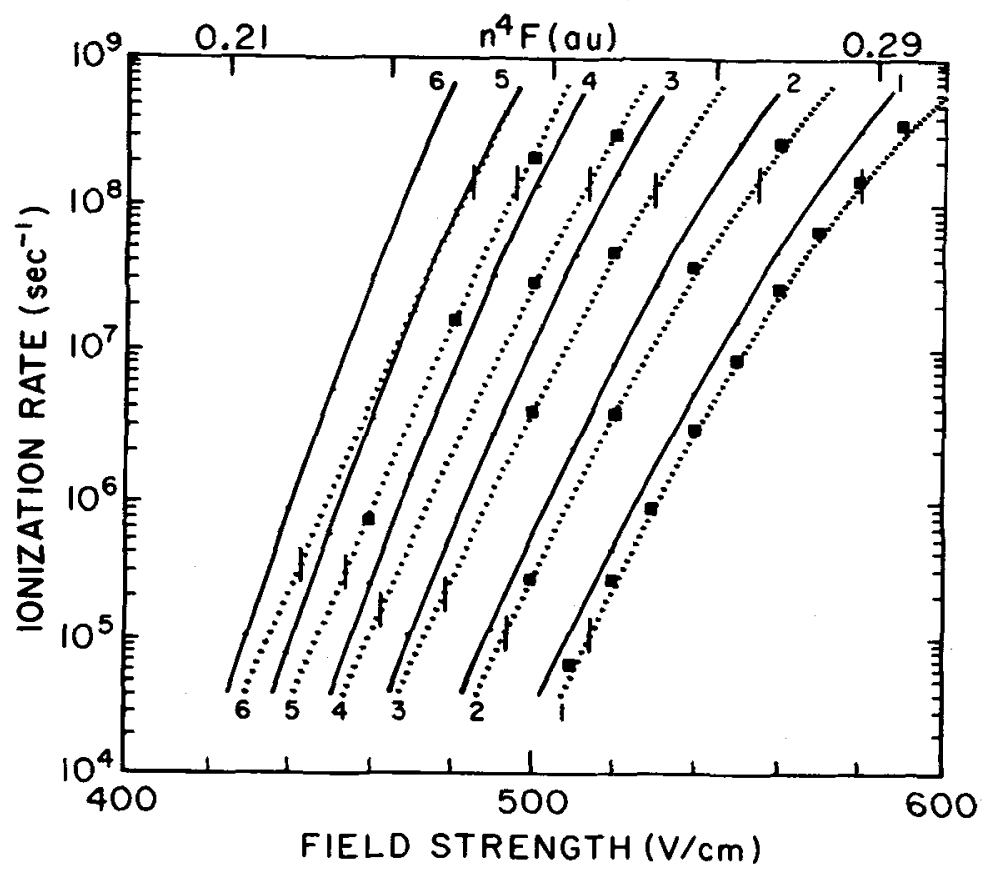

Fig. 3-Dotted lines: Experimental $\Gamma_{T}(F)$ curves for the hydrogen stark substates 1: $\{40,39,0,0\} ; 2:\{40,38,0,1\} ; 3:\{40,38,1,0\} ; 4:\{40,37,1,1\} ; 5:\{40,37$, $2,0\} ; 6:\{40,36,2,1\}$. The tic marks are discussed in Ref. 13. Solid lines: Theoretical curves calculated with use of a semiempirical, asymptotic formula (Eq. (6) of Ref. 14) with allowance for reduced-mass corrections. Squares: Numerical theoretical results calculated by Damburg and Kolosov for an infinitely massive nucleus.

These methods permitted the first precise measurement of the static electric field ionization rate $\Gamma_{I}(F)$ for resolved hydrogen stark substates. 13 Fig. 3 shows some experimental results (dotted lines) compared with numerical theoretical calculations (squares) and with calculations based on a semi-empirical analytic formula (solid lines) ${ }^{14}$. Since the six $n=40$ substates have large positive values of the "electric quantum number" $n_{1}-n_{2}=34 \rightarrow 39$, they ionize at relatively large values of the scaled field, $\mathrm{n}^{4} \mathrm{~F} \simeq 0.2-0.3$ au. Because of the quantal tunneling, ionization at rates $\simeq 10^{7} \mathrm{~s}^{-1}$ occurs at values of $\mathrm{n}^{4} \mathrm{~F}$ about $20 \%$ below the classical ionization thresholds calculated and displayed graphically by Banks and Leopold. I5 This behavior was also observed 3 for a state $(30,0,29,0)$ having a large negative value of $\left(n_{1}-n_{2}\right)=-29$. An important conclusion of this work is that quantal tunneling lowers the observable ionization threshold in static fields $(\omega=0)$ as much as $20 \%$ below the classical threshold.

4. Quantal vs. Classical Theories of Multiphoton Ionization. - It is an interesting and important question to determine whether tunneling would significantly lower the ionization threshold in an oscillatory field, $\omega \neq 0$. We are explicitly investigating this question in current experiments. Theoretically, the answer to this question is unclear. Keldysh ${ }^{16}$ introduced a semiclassical model that attempts to treat nonperturbatively tunneling and multiphoton ionization as different limiting cases of the same physical process, namely ionization of an atomic electron by an intense electromagnetic field. He introduced the use of a parameter $\gamma$ that is the ratio of 
the time required for the electron to tunnel through the instantaneous barrier to the period of the field; $\gamma$ much smaller and much larger than one correspond, respecitvely, to the adiabatic tunneling and multiphoton ionization limits. In atomic units, $\gamma=\omega / \mathrm{nF}_{\mathrm{o}}$ for hydrogen. Various assumptions made in his early work with this model were improved by Perelomov et al., 17 but it is not obvious that the model has any validity for the case of very high-order microwave ionization of $\mathrm{H}\left(\mathrm{high} \mathrm{n}\right.$ ) atoms. The large density of Rydberg states (proportional to $\mathrm{n}^{3}$ ) means that a large number of multiphoton resonances between intermediate states are possible on the way to the continuum; the model does not handle these. Nevertheless, a prediction of this model in the adiabatic limit $(\gamma<<1)$ is that ionization in the oscillatory field has a lower threshold than in a static field. As will be seen below, this is also a prediction of calculations based on classical theory, which ignores tunneling.

Other papers of this Colloquium by Brandi and by Mainfray deal with theoretical and experimental investigations, respectively, of the validity of the Keldysh model of ionization. Reiss 18 has also reinvestigated it theoretically. It has not been specifically applied to the problem of microwave ionization of atoms.

Leopold, Percival, and Jones (Refs. 6-8, see also a review of their work in another paper of this Colloquium by Richards) developed an entirely classical theory of microwave ionization and excitation. The theory depends on two parameters: (1) $\omega / \omega_{\text {at }}$, the ratio of the frequency of the field to the atomic orbital frequency, and (2) $\mathrm{n}^{4} \mathrm{~F}_{\mathrm{O}}$, the scaled peak electric field strength. In addition, they introduced the use of a "compensated energy" for the orbiting electron in the oscillatory field to decide whether ionization did or did not take place. From considerations of the magnitudes of classical actions compared to $\bar{h}$, they concluded that tunneling played a negligible role in the microwave experiments on $n=66$ atoms. 19,20 Since static ionization experiments show us that tunneling is very important in the $\omega=0$ limit, however, it is unclear at what values of the scaled frequency $\omega /$ wat and the scaled field strength $n^{4} F_{0}$ tunneling ceases to become important.

5. Typcial Parameters for Microwaves Used in the Experiments. - Microwave sources and plumbing span the range from $1 \mathrm{GHz}$ to more than $500 \mathrm{GHz}$. Generally, as the frequency goes up, the available output power of sources goes down, all components get smaller, and cost generally increases. There are a large number of microwave frequency bands; operation in a new band usually means that new components must be acquired. For this reason, the microwave experiments reviewed in this paper have not yet been performed at a large number of widely separated frequencies. Most have been in the range 7-12 GHz, where sources and components were available.

Table 1

\begin{tabular}{|c|c|c|c|c|c|c|c|c|}
\hline n & $F_{0}$ fo & $\mathrm{n}^{4} \mathrm{~F}_{\mathrm{o}}=0.1$ & $\mathrm{E} / \mathrm{h} \omega$ & $\Delta \mathrm{E}_{1} \psi^{\prime} \omega$ & $P_{\text {cav }}(W)$ & & $\mathrm{P}_{\mathrm{wg}}(\mathrm{W})$ & \\
\hline 10 & 51.4 & $\mathrm{kV} / \mathrm{cm}$ & 3292.1 & 658.0 & 10 & $\mathrm{~kW}$ & 3.1 & MW \\
\hline 20 & 3.2 & $\mathrm{kV} / \mathrm{cm}$ & 823.0 & 82.3 & 40 & W & 12 & $\mathrm{~kW}$ \\
\hline 30 & 0.6 & $\mathrm{kV} / \mathrm{cm}$ & 365.8 & 24.4 & 1.5 & $W$ & 0.4 & $\mathrm{~kW}$ \\
\hline 40 & 0.2 & $\mathrm{kV} / \mathrm{cm}$ & 205.8 & 10.3 & 0.2 & W & 0.05 & $\mathrm{~kW}$ \\
\hline 50 & 0.08 & $\mathrm{kV} / \mathrm{cm}$ & 131.7 & 8.8 & 0.03 & W & 7 & W \\
\hline 60 & 0.04 & $\mathrm{kV} / \mathrm{cm}$ & 91.4 & 3.0 & 0.006 & W & 2 & W \\
\hline 70 & 0.02 & $\mathrm{kV} / \mathrm{cm}$ & 67.2 & 1.9 & 0.002 & W & 0.5 & $\mathrm{~W}$ \\
\hline 80 & 0.01 & $\mathrm{kV} / \mathrm{cm}$ & 51.4 & 1.3 & 0.4 & $\mathrm{~mW}$ & 0.1 & W \\
\hline 90 & 8 & $\mathrm{~V} / \mathrm{cm}$ & 40.6 & 0.9 & 0.3 & $\mathrm{~mW}$ & 0.07 & W \\
\hline
\end{tabular}

Table 1 shows some useful numbers for $10 \mathrm{GHz}$ microwave ionization experiments of $\mathrm{H}$ (high $\mathrm{n}$ ) atoms. This frequency is near those used in most reported experiments. The columns show, respectively: (1) the principal quantum number, (2) the electric field strength $F_{0}$ needed to produce a scaled field strength $\mathrm{n}^{4} \mathrm{~F}_{\mathrm{o}}=0.1$ au, approximately the value observed empirically to produce appreciable ionization (notice that $\mathrm{n}^{4} \mathrm{~F}_{\mathrm{o}}$ is the classical field strength parameter mentioned above); (3) the ratio of the binding energy of the level to the $10 \mathrm{GHz}$ photon energy, or the number of photons energetically needed to get to the zero-field ionization limit; (4) the 
ratio of the energy separation between the levels $\mathrm{n}$ and $(\mathrm{n}+1)$, or the number of 10 $\mathrm{GHz}$ photons needed to drive a $\Delta \mathrm{n}=1$ transition (this ratio is also the inverse of the classical scaled frequency w/wat mentioned above); (5) the approximate power that must be coupled into (and dissipated in) a typical $\mathrm{TM}_{020}$-mode cylindrical cavity [unloaded $Q \sim 10^{4}$ and coupling voltage-standing-wave-ratio (VSWR) $\sim 1$ ] in order that the value of $F_{o}$ on the axis of the cavity equal that shown in column 2; (6) the approximate power that must be transmitted through a $\mathrm{TE}_{1}, 0$-mode $\mathrm{X}$-band (8-12 GHz), rectangular waveguide in order that the value of $F_{0}$ in the waveguide equal that shown in column 2 .

If we adopt $\sim 10 \mathrm{~W}$ as an affordable upper limit on the power available from a tunable, continuous microwave source (such as the combination of a low-power oscillator and a traveling-wave-tube amplifier), waveguide ionization experiments are restricted to $n \approx 50$ and cavity experiments to $n \geqslant 25$. This means that inter $-n$ transitions require on the order to ten photons, whereas ionization requires on the order of one hundred photons. Higher power, fixed frequency sources such as magnetrons are available in this and higher frequency ranges, but they would be best suited to experiments using resonant cavities. With them, one would be unable to study the frequency dependence of atom-field interactions. As we shall see, such studies are extremely important and have uncovered resonant behavior that is only partially understood.

6. Previous Ionization Experiments and Some New Results. - Bayfield and Koch ${ }^{19}$ first reported eight years ago the observation of microwave ionization of $\mathrm{H}$ (high $\mathfrak{n}$ ) atoms in the n-band $63 \leqslant n \leqslant 69$. Fig. 4 shows their data for the ionization probability at $9.91 \mathrm{GHz}$ (solid circles), which corresponds to a scaled frequency $\omega / \omega_{a}=0.43$. The atoms adiabatically entered and exited the microwave field inside a $\mathrm{TM}_{020}$-mode cavity and were exposed to about $10^{3}$ oscillations. The open circles show the classical, Monte-Carlo theoretical results of Leopold and Percival.6,7 Since, to date, these are the only calculations in quantitative agreement with the ionization experiments, the classical theory must be examined further as a viable model for the interaction of Rydberg atoms with intense microwaves. The horizontal axis is in units of the parameter $\gamma$, which in the classical theory is the ratio $\left(\omega / \omega_{a t}\right) /$ $\left(\mathrm{F}_{\mathrm{O}} / \mathrm{F}_{\mathrm{at}}\right)$ of the classical frequency and field strength parameters. It is numerically equal to the Keldysh parameter, but its interpretation is very different because the classical theory obviously does not account for tunneling ionization.

It was found ${ }^{6-8}$ that the classical electron orbits in the intense field could be grouped into four different classes Cl-C4. Each could be recognized by its distinctive spatial trajectory or by a plot of its "compensated energy" $E_{c}$ vs. time. The latter are shown in Fig. 5. Motions $\mathrm{Cl}$ lie on an invariant torus in phase space and may never ionize; C2 ionize rapid1y and directly into the continuum; C3 ionize more slowly and pass through at least one intermediate, extra-highly-excited (EHE) state; $\mathrm{C} 4$ pass through one or more EHE but do not ionize during the time scale of the calculation. Notice that $\mathrm{C} 3$ and $\mathrm{C} 4$ exhibit de-excitation (downward) transitions as well as excitation (upward) transitions.

The classical, non-linear dynamics of the ionization process is complicated. It is not clear how one would construct a quantum mechanical theory of microwave ionization that would reproduce this behavior and give physical insight into the different types of electronic motions. Stable and unstable (escaping) classical trajectories may co-exist for different initial conditions at the same values of the classical parameters, and both excitation and de-excitation transitions seem to be important. It appears from the plots in Fig. 5 that the electron may not end up with much kinetic energy after ionization, but this was not explicitly evaluated in the classical calculations. 6-8 With use of a strong-field, quantal, perturbative theory, Gersten and Mittleman 21 attempted to estimate how far into the continuum the electron would be ionized, but the validity of their theory for the microwave experiments was very uncertain. They tentatively concluded that, on average, twice the minimum number of photons would be absorbed. Since the Rydberg atoms in the experiments were bound by only tens of meV, their result, if true, means that the ionized electrons would have only this amount of kinetic energy. Recent laser multiphoton ionization experiments (reviewed in another talk of this Colloquium by Mainfray) have determined the relative probability for ionization of atoms by more than the minimum number of photons, but the theoretical interpretation of these 


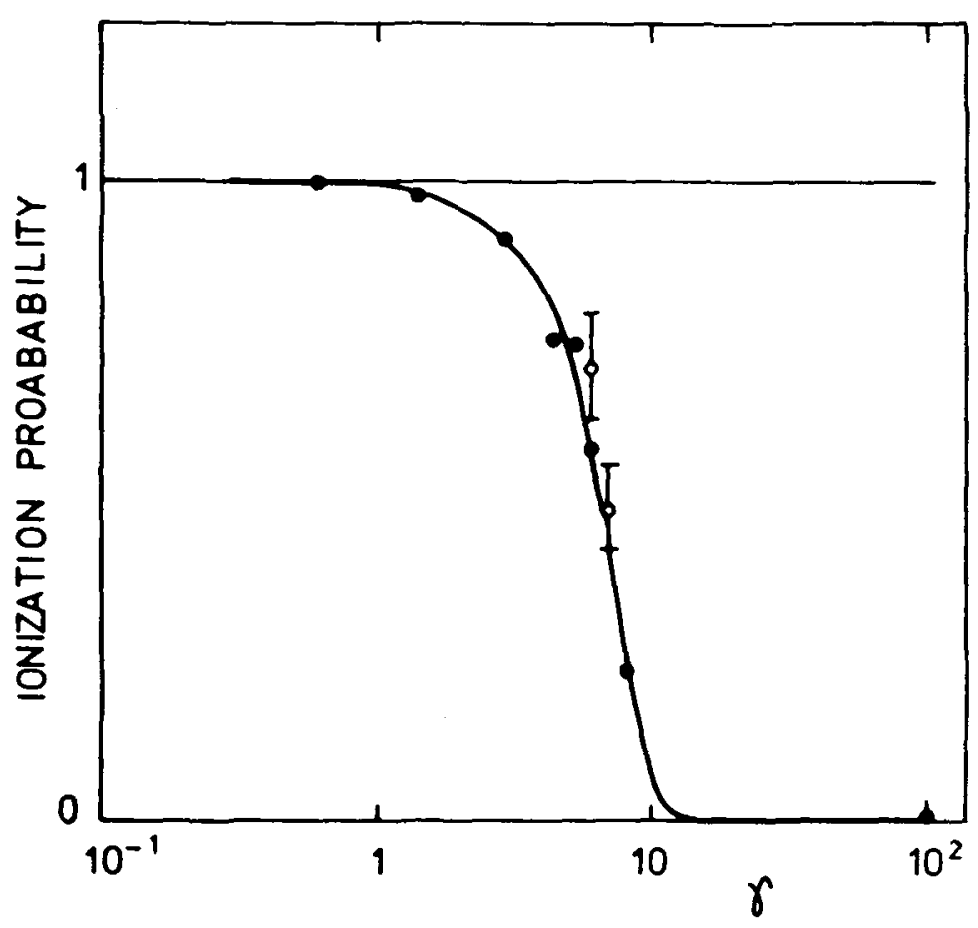

Fig. 4 - Closed circles: The measured probability (Ref. 19) for ionization of $\mathrm{H}$ (high $\mathrm{n}$ ) atoms in the $\mathrm{n}$-band $63<\mathrm{n}<69$ by a $9.91 \mathrm{GHz}$ microwave field as a function of the parameter $\gamma$, which is numeriżcally equal to both the "Keldysh" parameter (Ref. 16) used in a quantal theory and to the ratio $\left(\omega / \omega_{a t}\right) /\left(F_{\circ} / F_{a t}\right)$ of the two parameters used in a classical theory (Refs. 6-8) of the interaction of hydrogen atoms with an oscillatory electric field. Open circles: Results of Monte-Carlo calculations (Refs. 6,7 ) that employed the classical theory to model the experiment of Ref. 19.(taken from Ref. 6)

experiments is still in progress.

It may be possible to use electron spectroscopy to gain information about the distribution of kinetic energy of electrons ionized by microwaves. Showing the feasibility of this type of measurement was a by-product of a very recent collaborative experiment at Argonne National Laboratory undertaken by our Yale group and a group at the Argonne Dynamitron Laboratory. More will be said at the end of this article about the experiment, whose data are still being analyzed.

7. Frequency-dependent Effects.- The resonant response of $\mathrm{H}$ (high $\mathrm{n}$ ) atoms to certain ranges of microwave frequency was observed by Bayfield, Gardner, and Koch ${ }^{20}$. In order to be able to sweep the frequency over the range $09.6-11.4 \mathrm{GHz}$, the atom beam was directed through an $\mathrm{X}$-band, $\mathrm{TE}_{1,0}$ mode waveguide. As was explained earlier via Table I, available microwave powers $\underset{\leqslant}{\leq} 20 \mathrm{~W}$ meant that ionization of atoms 1 aser excited into individual $\mathrm{n}$-levels could be studied for $\mathrm{n}<45$. Fig. 6 shows ionization curves for each level between $n=45$ and $n=57$. Fig. 7 shows, just for $n=48$, an ioni-zation curve (lower frame) and an excitation curve (upper frame). The latter was taken in a "two microwave field" experiment that was sensitive only to atoms that were excited to $n>48$ bound levels, but not to the continuum. Both curves in Fig. 7 were taken at a microwave power of $8 \mathrm{~W}$, which corresponds to $\mathrm{F}_{0} \simeq 80 \mathrm{~V} / \mathrm{cm}$. Each curve 

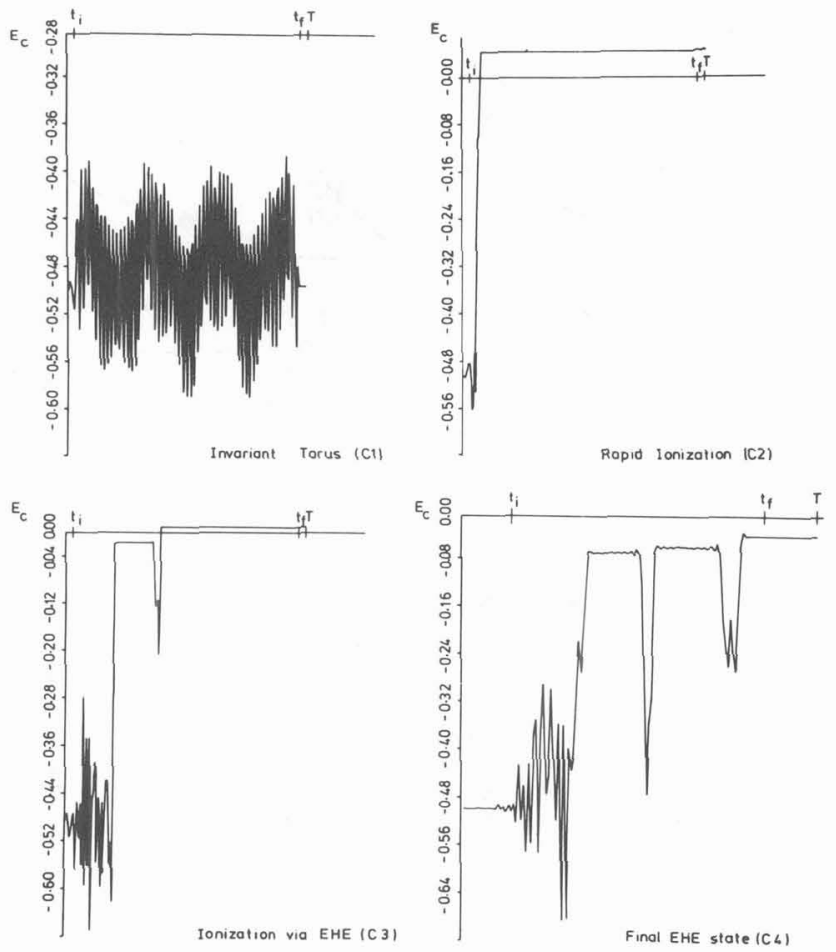

Fig. 5 - Plots of the "compensated energy" $E_{c}$ vs. time for four different types of orbits C1-C4 obtained with Monte-Carlo calculations (Refs. 6-8) that were based on a classical theory of the interaction of hydrogen atoms with an oscillatory electric field. The calculations followed the electron during several hundred field oscillations. When $E_{c}<0$, the electron is still bound to the proton. When $E_{c}>0$, the electron is ionized. EHE stands for an extra-highly-excited Rydberg state with principal quantum number about a factor of five larger than the initial one. (taken from Ref. 6)

in Fig. 6 was recorded at a different microwave power empirically adjusted to limit the fraction of atoms ionized to 1-30\%. The data in both figures lie in the following ranges of the classical parameters: (1) $0.13<\omega / \omega_{0} \leq 0.26$ (or in the nearadiabatic frequency regime), and (2) $\mathrm{F}_{\mathrm{o}} / \mathrm{F}_{\text {at }}=\mathrm{n}^{4} \mathrm{~F}_{\mathrm{o}} \simeq 0.09 \mathrm{au}$, or significantly below the classical ionization thresholds $\mathrm{F}_{\mathrm{o}}^{\mathrm{a}} / \mathrm{F}_{\text {at }}=0.13-0.38 \mathrm{au}^{8,15}$.

The $\leadsto 8 \mathrm{~mm}$ diameter beam-entrance and -exit holes perturbed the waveguide electric field distribution so that $F_{0}$ was within $25 \%$ of its peak value only over a fraction, perhaps half or less, of the $1 \mathrm{~cm}$ separation between the opposing faces. This meant that the $\sim 10 \mathrm{keV}$ atoms experienced a transit-time-induced frequency spread of $0.05 \mathrm{GHz}$ or more. The $0.5-1.0 \mathrm{GHz}$ widths of the resonances in Figs. 6 and 7 are significantly larger than this, so they may be physically important. The absolute rate scales in Fig. 7 show that multiphoton ionization and excitation may proceed at comparable rates; which is dominant depends sensitively on the frequency.

These experimental results stimulated theoretical activity. Delone et a1.11 advanced a diffusion model to characterize the microwave ionization, but their 


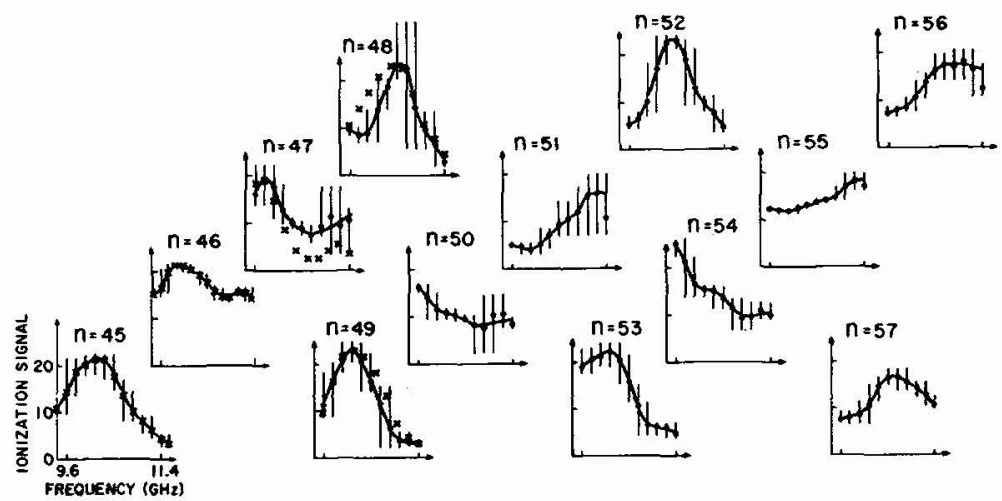

Fig. 6- The measured ionization of $\mathrm{H}(\mathrm{high} \mathrm{n})$ atoms with each principal quantum number $n$ between 45 and 57 as a function of the microwave frequency between 9.6 and $11.4 \mathrm{GHz}$. The vertical scales are relative units different for each $\mathrm{n}$. The vertical bars are worst-case estimates of the fluctuations in the signals that were caused by frequency-dependent variations in the peak microwave electric field strength experienced by the atoms inside the $\mathrm{TE}_{1}, 0$ 'mode waveguide. The microwave power incident on the waveguide was kept approximately constant for each n-value but was readjusted with each change in $n$ to keep the peak fractional ionization in the range $1-30 \%$.

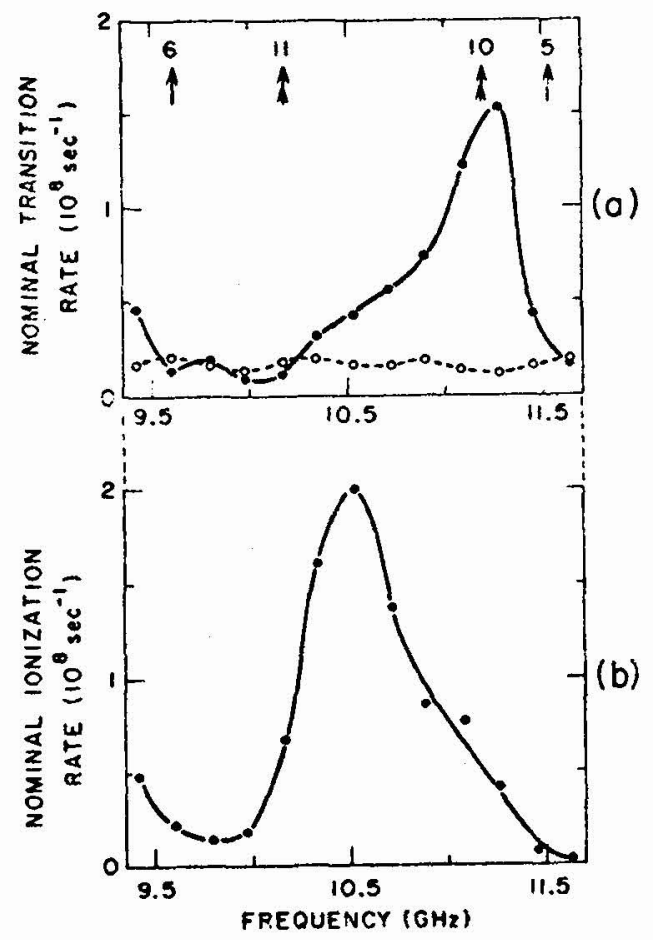

Fig. 7- A comparison of the frequency dependence and absolute rates for microwave multiphoton excitation (a) and ionization (b) of $\mathrm{H}(\mathrm{n}=48)$ atons. The open circles in (a) are background signals. Single- and doubleheaded arrows show the position of multiphoton transition frequencies between a field-free $\mathrm{n}=48$ state and field-free $\mathrm{n}=49$ and $n=50$ states, respective $1 y$, for the number of photons shown above the arrows. 
estimated diffusion time was criticized by Meerson et a1. 10 , who proposed an ionization model based on the stochastic instability of a classical, non-linear oscillator. In pointing out that their own classical, Monte-Carlo trajectory results were directly related to the general problem of the onset of stochastic motions in non-linear dynamical systems, Percival et a1.6-8 concluded that "much more work needs to be done...to obtain a clear picture of the physical process responsible for ionization." In a recent review article Zaslavsky amplifies and extends these points of view and "...show(s) the possibility of the existence of a stochastic mechanism for the ionization of atoms." (Ref. 9, p.236).

A possible, more fundamental problem concerns the nature of stochasticity in quantum systems. Again quoting Zaslavsky (Ref. 9, p.160):

At present, the number of papers considering stochasticity in quantum systems is rather large. Not only is there no understanding of the general situation but there is no more or less clear answer to even a single, the "simplest", problem, either. Among them are the fact that not even a single model has been accurately solved completely, that there are no exact results, that there is the necessity to obtain results true for long times, and that there are difficulties in using computers.

Zewail concludes, similarly, that the underlying theoretical is "...the question of what precise relationship, if any, exists between classical chaos and quantum mechanical motion. Clearly we still do not know the precise meaning of quantum ergodicity." (Ref. 22, p.33).

Since the classical and quantum mechanical motions of the field-free hydrogen atom are known exactly, the hydrogen atom in an electromagnetic field may be a useful testing ground for intense-field studies of these issues. One of them is obviously what influence quantal tunneling may have on the onset of chaotic and escaping motions in non-linear systems.

8. New Hydrogen and Helium Experimental Results.- At Yale, our group has recently completed running some new experiments on microwave ionization of hydrogen and helium Rydberg atoms. The goals were rather modest: We were interested in comparing the $\mathrm{F}_{\mathrm{o}}$-dependence of the ionization of a simple non-hydrogenic atom with that of hydrogen. The apparatus was similar to that shown in Figs. 1a,b, and a $9.91 \mathrm{GHz} \mathrm{TM}_{02} 0^{-m o d e}$ cavity was used to produce the ionizing microwave field. Analysis of these new experiments is still in progress, and the author appreciates the willingness of his colleagues at Yale, D. Mariani and $W$. van de Water to have some of our preliminary results and conclusions presented here.

For a variety of reasons, we chose to work with $n^{3} S_{1}$ Rydberg states of helium. We shall compare results for atoms in different n-manifolds by using the scaled electric field $n * 4 F$, where $n *=[n-\delta(L, S)]$, the effective quantum number, equals the difference of the principal quantum number $n$ and the quantum defect $\delta(L, S)$, which depends mainly on the orbital $L$ and spin $S$ angular momenta of the particular level. Helium $n^{3} S_{1}$ Rydberg levels have the largest quantum defect, $\delta(0,1)=0.297$. Since it will be useful to compare their microwave ionization behavior to that in a static field, we show in Fig. 8 a static field ionization curve for $\mathrm{He}\left(41{ }^{3} \mathrm{~S}_{1}\right)$ atoms prepared by double-resonance, $\mathrm{CO}_{2}$ laser techniques $2,12,13$ in fields $\mathrm{F}_{1}-\mathrm{F}_{5}$ in $\mathrm{Figs}$. $1 \mathrm{a}, \mathrm{b}$. Fig. 8 shows the fraction of an $012 \mathrm{keV}$ beam of these atoms that survived passage through the $8.3 \mathrm{~cm}-1$ long ionizing field $F_{5}$ in Fig. la. It was recorded using the same methods previously used for precise measurement of field ionization rates of hydrogen Stark substates ${ }^{3}$. We notice a sharp ionization threshold (drop in the curve) at $n * 4 F \simeq 1 / 16$ au, which is the "saddle-point" ionization theshold that characterizes ionization of non-hydrogenic, low- $|\mathrm{m}|$ Rydberg states that follow adiabatic paths to the continuum ${ }^{23}$.

Fig. 9 shows microwave ionization curves for $H(n=41)$ atoms (with an unmeasured distribution of substates) and $\mathrm{He}\left(41^{3} \mathrm{~S}_{1}\right)$ atoms. Each curve shows a signal proportional to the fraction of atoms ionized during their passage through the cavity; 

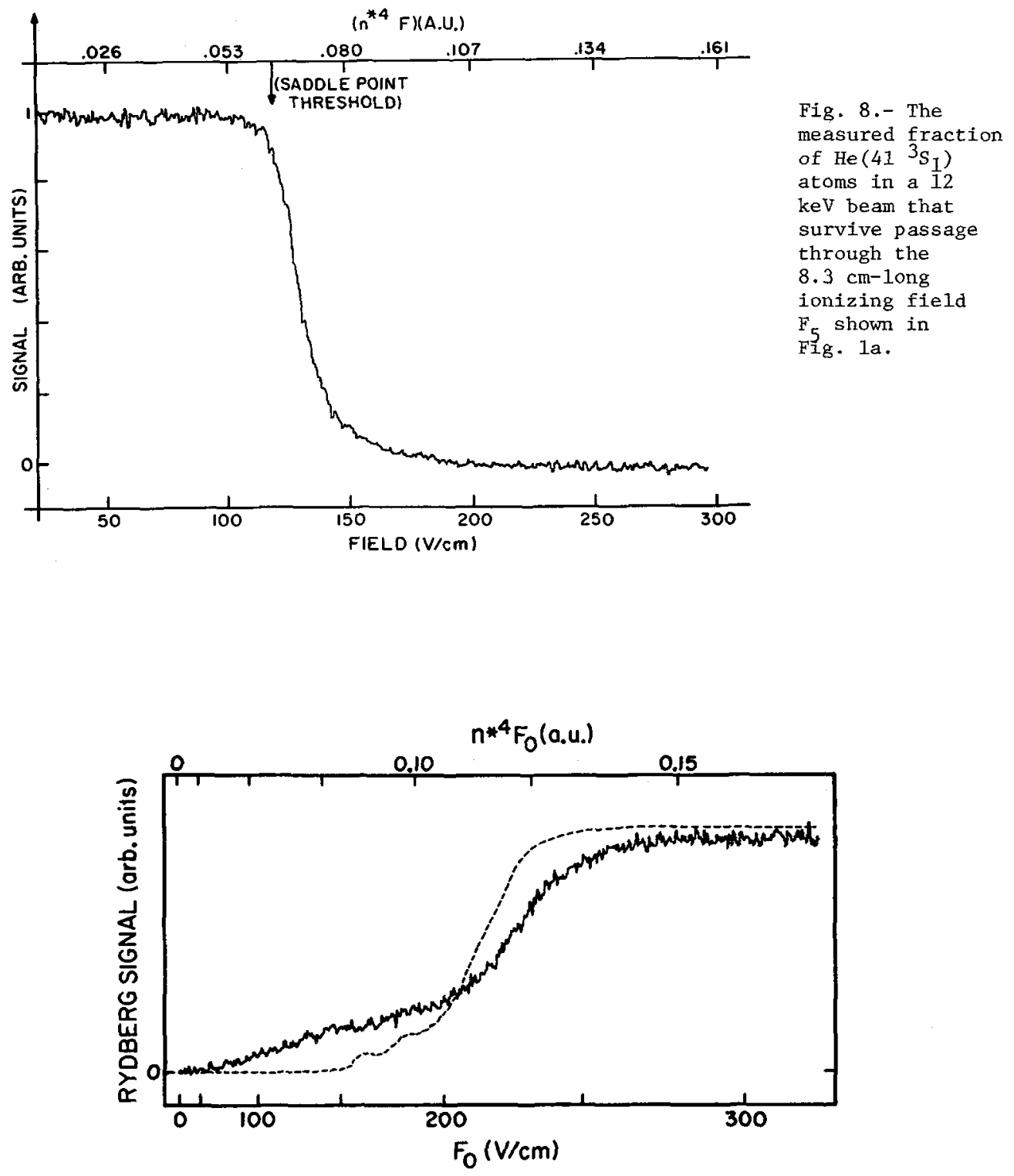

Fig. 9 - Microwave ionization curves for $H(n=4 I)$ atoms (dashed curve) and He $\left(41^{3} \mathrm{~S}_{1}\right)$ atoms (solid curve). The horizontal scale is proportional to the microwave power incident on the $9.91 \mathrm{GHz}$, TM $00^{-m o d e}$ cavity; therefore; the scale of the peak amplitude of the microwave electric field $F_{0}$ is non-linear. The absolute $\mathrm{F}_{\mathrm{O}}$-scale is uncertain by about $10 \%$. The $\mathrm{H}$ and $\mathrm{He}$ atoms were exposed to about $6.5 \times 10^{2}$ and $1.3 \times 10^{3}$ cycles of the microwave field, respectively. The signals saturate for $n{ }^{4} \mathrm{~F}_{\mathrm{o}} \gtrsim 0.12-0.14$ au. 
the two curves have been approximately normalized. For $n \simeq 41$, the $9.91 \mathrm{GHz}$ frequency corresponds to a frequency ratio $\omega / \omega_{a t} \simeq 0.1$, or in the "near-adiabatic" regime. Even though the difference in zero-field binding energies of the atoms is very small, $\Delta \mathrm{E} \approx \delta(0,1) / \mathrm{n}^{3}$ au $=0.95 \mathrm{~cm}^{-1}=28 \mathrm{GHz}$, or fractionally only $1.4 \%$, the two ionization curves are very different. The hydrogen ionization threshold is approximate $1 \mathrm{y} \mathrm{n}^{4} \mathrm{~F}=0.08$ au, and there appears to be some structure in the curve near threshold. The ionization signal is saturated (rate $>10^{8} \mathrm{~s}^{-1}$ ) at $\mathrm{n}^{4} \mathrm{~F}_{\mathrm{o}} \simeq 0.13$ au. For helium, however, the ionization signal rises at much smaller values of $n^{4} F_{0}$ and is not saturated until a somewhat higher value than that for hydrogen. Fig. 10 shows another hydrogen curve, this time for $n=27$. For this $n-$ level, the frequency ratio is $\omega / \omega_{0 t} \simeq 0.03$, or even more into the adiabatic regime. In terms of the scaled field $n^{4} F_{0}$, the $H(n=27)$ curve is very similar to the $H(n=41)$ curve, except for the lack of possible structure near threshold.

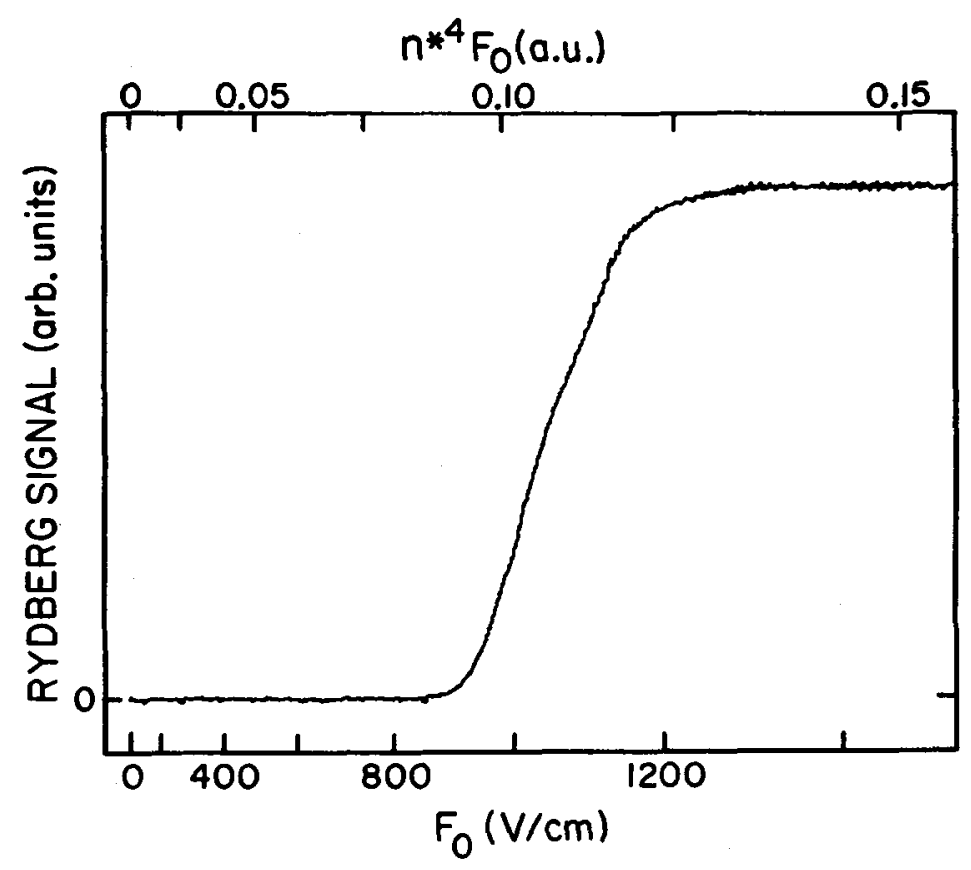

Fig. 10 - A microwave ionization curve for $H(n=27)$ atoms that was taken under the conditions described in the caption to Fig. 9. The signal saturates for $\mathrm{n}^{4} \mathrm{~F}_{\mathrm{o}}>0.12 \mathrm{au}$.

Let us analyze the hydrogen curves first in terms of the classical, Monte Carlo theoretical results ${ }^{8}$. Fig. 11 shows, for a number of values of the scaled field $F_{O} / F_{\text {at }}=n^{4} F_{O}$, a composite of their calculations of the percentage of ionization vs. the frequency ratio $\omega / \omega$. (We point out that more extensive calculations at fixed $\mathrm{F}_{\mathrm{o}} / \mathrm{F}_{\text {at }}=0.08$ and cn a finer mesh of frequency ratios $\omega / \omega_{\text {at }}$ between 0.3 and 1.4 shows several resonances in the ionization probability (Ref. 8 , Fig. 3) not unlike those observed experimentally--see Figs. 6,7 above. Unfortunately this range of $\omega / \omega$ at is slightly above the range $0.13-0.26$ of the experiments 20 .) We notice that for $\omega / \omega_{\text {at }}<0.1$ (or to the left of $\lg \left(\omega / \omega_{\text {at }}\right)=-1$ on each horizontal scale), they predict that negligible ionization takes place until $F_{0} / F_{\text {at }}>0.1$. Recall that the measured hydrogen curves in Figs. 9, 10 show the onset of ionization signals at $\mathrm{F}_{\mathrm{o}} / \mathrm{F}_{\text {at }} \simeq 0.08$. A preliminary analysis leads to about a $10 \%$ uncertainty in our determination of the absolute field strength $\mathrm{F}_{\mathrm{o}}$ in the cavity in terms of the measured power incident on it. Therefore, we have some preliminary evidence that the observed onset of ionization occurs at somewhat lower values of $F_{0} / F_{\text {at }}$ than those predicted by the classical theory $6-8$. It is highly tempting to view this as evidence for the importance of tunneling, but we shall avoid this categorical assertion until we have made a complete analysis of our data and until a larger 


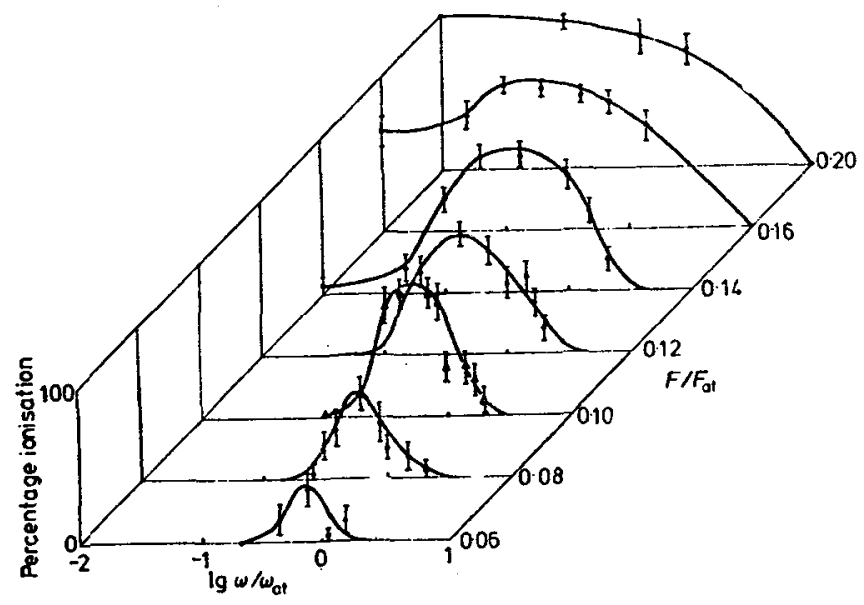

Fig. 11 - A composite of the results of classical Monte-Carlo calculations (Ref. 8) at a number of values of the scaled field $F_{0} / F_{\text {t }}$ of the percentage ionization of hydrogen atoms in an oscillatory electric field whose frequency $\omega$ is related to the atomic orbital frequency through the ratio $\omega / \omega_{\text {at }}$. Notice that the ionization threshold for a nearly static field is near $\mathrm{F}_{\mathrm{o}} / \mathrm{F}$ at $=0.13$ au and that the ionization threshold for oscillatory fields with $\omega / \omega_{0}$ at 1 is about a factor of two lower. The classical calculations ignore the effects of quantal tunneling, which lowers the observed static ionization threshold (Ref. 13) by 10-20\% depending on the particular Stark substate studied. (taken from Ref. 8)

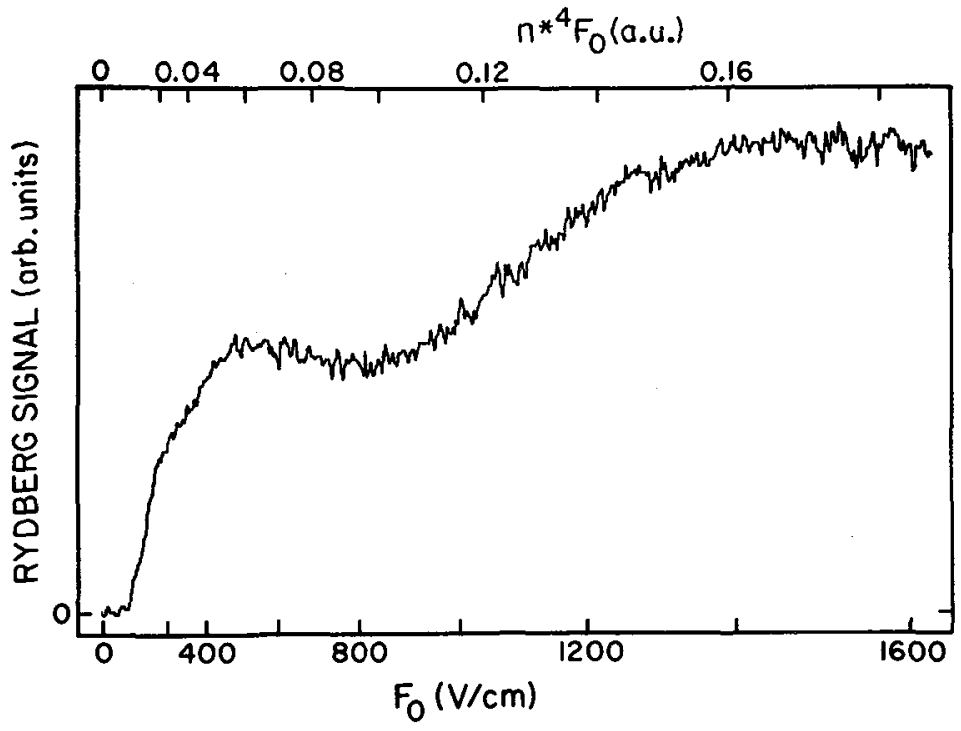

Fig. 12- A microwave ionization curve for $\mathrm{He}\left(28{ }^{3} \mathrm{~S}_{1}\right)$ atoms that was taken under the conditions described in the caption to Fig. 9. The signal saturates for $\mathrm{n} * 4 \mathrm{~F}_{\mathrm{o}}>0.16$ au. 
number of classical calculations are available in the threshold region.

Now we move to a discussion of the helium microwave ionization data. Fig. 12 shows an ionization curve for $\mathrm{He}\left(28{ }^{3} \mathrm{~S}_{1}\right)$ atoms. Similar to the $\mathrm{He}\left(41{ }^{3} \mathrm{~S}_{1}\right)$ curve shown in Fig. 9, the ionization signal rises at very low values of $n * 4 \mathrm{~F}_{0}$, but the $\mathrm{He}\left(28{ }^{3} \mathrm{~S}_{1}\right)$ curve has a plateau (and even a slight dip) between about $\mathrm{n}^{* 4} \mathrm{~F}_{\mathrm{o}}=$ 0.05-0.1 au, rises again above this value, and does not saturate until about $0.16 \mathrm{au}$. Fig. 13 shows an ionization curve for He $\left(28{ }^{3} \mathrm{~S}_{1}\right)$ over a much smaller

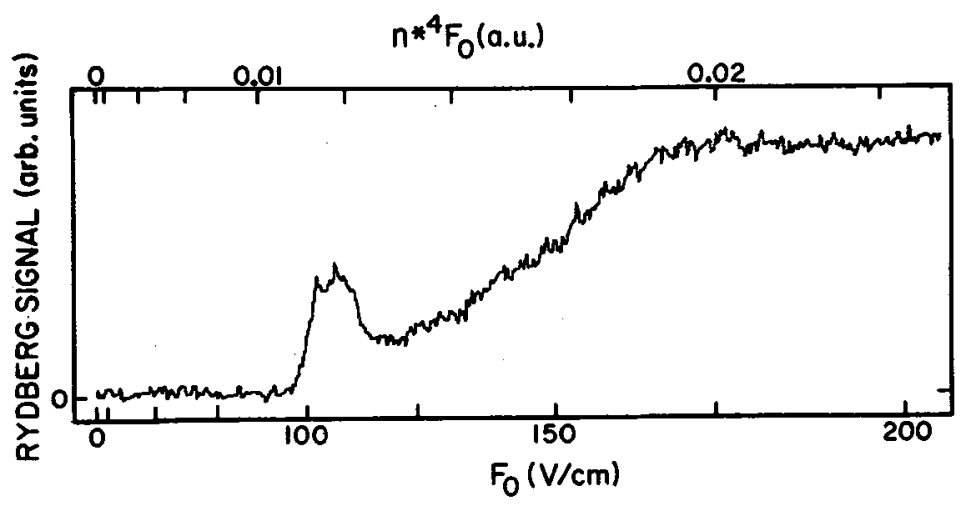

Fig. 13 - A microwave ionization curve for He $\left(28{ }^{3} \mathrm{~S}_{1}\right)$ atoms that emphasizes the threshold, 10w-F region of Fig. 12. The origin of the "bump" is explained in the text. The signal for $\mathrm{n}^{4} \mathrm{~F}_{\mathrm{O}}>0.02$ au is not saturated; it is a plateau unobservable on the scale of Fig. $\tilde{6}$.

range of $n * 4 \mathrm{~F}_{0}$-values near the onset of ionization. Notice that $\mathrm{F}_{\mathrm{o}}$ scales of the two figures are so different that the very interesting threshold structure observable in Fig. 13 is compressed and unobservable on the scale of Fig. 12. We see that the ionization signal in Fig. 13 begins very sharply at $n * 4 F_{0} \simeq 0.011$ au, or $\mathrm{F}_{0} \approx 100 \mathrm{~V} / \mathrm{cm}$, rises to an local maximum, decreases, rises again more slowly, and then reaches a local plateau.

We are able to explain the "bump" near $100 \mathrm{~V} / \mathrm{cm}$ in terms of known features of the helium spectrum. Fig. 14 shows a "Stark map" of m=0 triplet Rydberg levels. The calculation was performed on an $\left(n, \ell, m_{\ell}\right)$ basis by $D r$. van de Water of our group using matrix-diagonalization programs adapted from those kindly supplied by Dr. Zimmerman ${ }^{24}$. The magnetic fine-structure of helium is negligible on the scale of the map and was ignored in the calculation. Notice that the adiabatic continuation of the He $\left(28^{3} \mathrm{~S}_{1}\right)$ level undergoes its first avoided crossing with the uppermost $m=0$ triplet level (which is the adiabatic continuation of the $(n=27, \ell=26$, $\mathrm{m}_{\ell}=0$ ) leve1) rising from the $\mathrm{n}=27$ manifold near $110 \mathrm{~V} / \mathrm{cm}$, which is within estimated experimental error of the position of the threshold "bump" in the ionization curve. Since the frequency ratio $\omega / \omega \simeq 0.03$, the oscillation of the applied electric field is adiabatic with respect to the motion of the electron. The "Stark map" in this case is analogous to the molecular potential curves that one obtains in a Born-Oppenheimer separation of the slow internuclear motions from the fast electronic motions in a molecule. In a molecule, or in a quasi-molecular treatment of an atom-atom collision, the internuclear motion produces dynamic terms which couple states belonging to different adiabatic molecular potential curves. In the present case, the oscillatory field moves the system back and forth along the Stark potential curves and may cause the system to jump (make diabatic, or Landau-Zener transitions ${ }^{25}$ from one potential curve to another. 


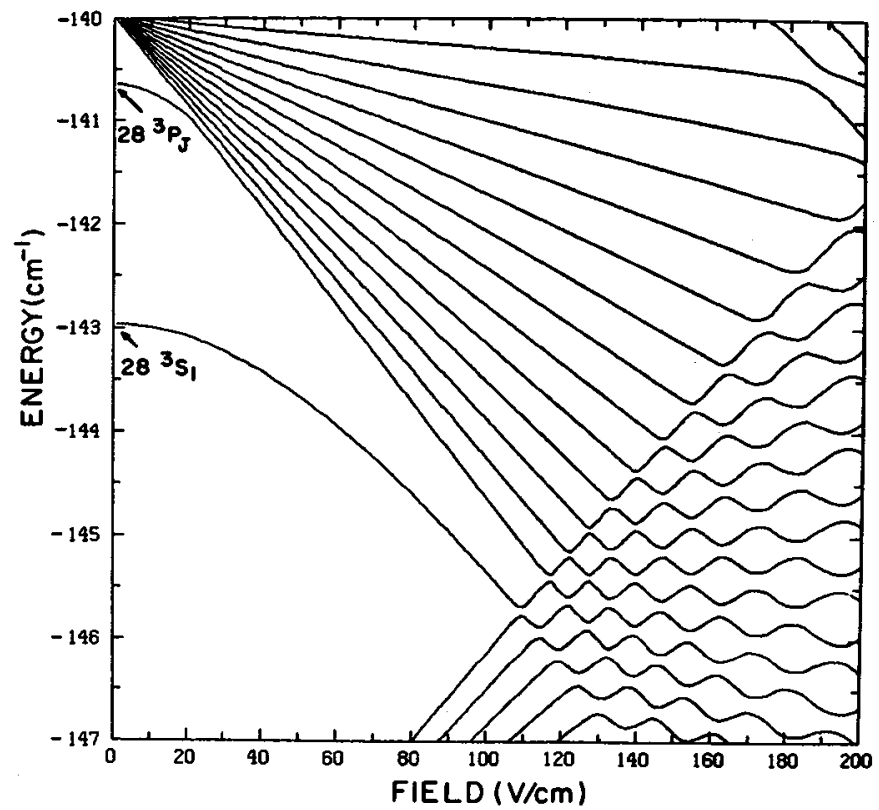

Fig. 14- A preliminary "Stark map" for some m=0 triplet Rydberg levels of helium that was calculated by $\mathrm{Dr}$. W. van de Water at Yale with use of matrix-diagonalization programs adapted from those kindly supplied to us by Dr. M.L. Zimmerman (Ref. 24). It was computed on an $\left(n, l, m_{l}\right)$ basis of 106 states in the $n=26-29$ manifolds. Magnetic fine-structure is unobservable on the scale of the figure and was neglected.

It appears, therefore, that partially diabatic transitions at this first and at other avoided crossings transfer population from the initial level onto other Stark substates. The remarkable thing is that the system is then able to proceed all the way to the continuum at this rather low field strength. Since the atom experienced approximately $10^{3}$ oscillations of the field, however, it had many opportunities to make such transitions. We note that the first avoided crossing between adiabatic levels in neighboring manifolds, $n$ and ( $n-1)$, occur at an $F_{0}-$ value that scales as $n^{-5}$.

In additional experiments with $\mathrm{n}=26,29 \mathrm{He}\left(\mathrm{n}{ }^{3} \mathrm{~S}_{1}\right)$ atoms, we observed threshold "bumps" and "plateaus" similar to those shown for n=28 in Figs. 6,7. For these other n-values, which also have adiabatic frequency ratios $\omega / \omega$, the bumps show up at $F_{0}$-values corresponding to the first avoided crossing. We did not observe a threshold "bump" with He(41 $\left.{ }^{3} \mathrm{~S}_{1}\right)$ atoms, however. It is tempting to explain this behavior by the Iess adiabatic frequency ratio $\omega / \omega_{\text {at }} \simeq 0.1$ for $n=41$. If this is so, one would have an effect analagous to the breakdown of the Born-oppenheimer adiabatíc approximation in molecules.

Though our preliminary analysis explains, at least partially, the origin of some threshold bumps, we cannot explain quantitatively the location of plateau regions over which some ionization curves are locally independent of the value of $\mathrm{F}_{\mathrm{O}^{*}}$ Our experiments on microwave ionization of non-hydrogenic atoms have displayed fascinating behavior that is very different from that observed in hydrogen atoms having nearly the same initial binding energy. One may speculate that the hydrogen-like, high- $|\mathrm{m}|$ states of a Rydberg atom like helium may display microwave ionization behavior more like that of hydrogen. Since the electric field interaction conserves $\left|\mathrm{m}_{\ell}\right|$ (at least in the limit of negligible magnetic fine-structure), 
the oscillatory electric field would not induce transitions between different $\left|m_{\ell}\right|-$ states. Stray fields or collisions could do this, however.

We offer an important conclusion: Nearly all the theories treating microwave ionization have been based on a non-relativistic treatment of hydrogen. Since we have observed dramatic differences between hydrogen and non-hydrogenic stark states, it is inappropriate to use non-hydrogenic Rydberg atoms in experiments designed to test theories based on hydrogen. Nevertheless, the non-hydrogenic effects will be interesting to study in detail in their own right, but one will need to develop additional theory.

9. Structure of $\mathrm{H}$ (high $\mathrm{n}$ ) Atoms in an Intense Microwave Field.- Thus far, we have emphasized questions of atomic transitions driven by intense microwaves, from one bound state to another or from a bound state to the continuum. In this section, we consider the problem of atomic structure, or what are the (quasi-) bound energy levels of the hydrogen atom in the intense microwave field. Blochinzew ${ }^{26}$ first investigated the quantal structure of the Hamiltonian Eq. (2). More recent treatments of the "AC-Stark effect" have included those of Autler and Townes ${ }^{27}$, of Bakshi et $a 1.28$, of Hicks ${ }^{29}$ and Hicks et al. ${ }^{30}$, and of a number of other theorists whose work has been reviewed recently by Delone et al. ${ }^{31}$ and by Bayfield ${ }^{4}, 5$. For the degenerate states of the hydrogen atom, these theories predict that one effect of an intense, linearly polarized oscillatory field is to produce quasi-energy satellites (also called dressed states, phot on replicas, etc.) displaced by integral numbers of photons \pm phw above and below the zero field level. The amplitude of the satellite of order $\mathrm{p}$ is proportional to

$$
J_{p}\left(d F F_{0} / x_{L} \omega\right),
$$

the value of the Bessel function of order $p$ whose argument is the ratio of the peak dipole energy $d F_{0}$ to the photon energy $h \omega$. As in the static Stark effect, the permanent dipole moment $d$ is created from opposite-parity atomic states that are mixed by the field. The Bessel function weighting of the satellites is a general feature of intense-field problems: they appear, for example, in spin systems in intense, oscillatory magnetic fields ${ }^{32}$ and in electron-atom collisions in the presence of an intense laser field ${ }^{33}$.

Twenty years ago, Baranger and Mozer ${ }^{34}$ proposed using spectral-1ine-satellites produced by the AC-Stark effect as a diagnostic probe of high-frequency electromagnetic oscillations in a plasma. This motivated a significant amount of experimental and theoretical work (for useful lists of references see Refs. 35 and 36). The Ph.D. thesis of Hicks ${ }^{29}$ includes the report of a nice optical spectroscopy experiment on a hydrogen plaswa, but to this author's knowledge, it is available only as a laboratory report. He measured the Balmer spectrum emitted by the hydrogen plasma inside a Geissler tube that was collinear with the microwave electric field inside a rectangular, $\mathrm{TM}_{010}$-mode cavity. He was able partially to resolve the microwave-induced satellites on each side of the $\mathrm{H}_{\alpha}, \mathrm{H}_{\beta}, \mathrm{H}_{\gamma}, \mathrm{H}_{\delta}$ lines and to obtain excellent results with a theory based on the work of Blochinzew.

The agreement between experiment and Blochinzew-type theory has generally been much less impressive for experiments which used internal fields inside a plasma, particularly a turbulent one, to produce the satellites. An externally applied field was used in the published helium experiment of Hicks et a1. ${ }^{30}$ and in the hydrogen experiment just mentioned ${ }^{29}$. Nee and Griem ${ }^{35}$ observed internal-plasma-fieldinduced $p= \pm 1$ satellites of $n=13 \rightarrow 12$ and $n=12 \rightarrow 11$ infrared transitions in hydrogen but found that the absence of higher-order satellites could not be explained by a Blochinzew-type theory based on a single-frequency, constant amplitude electric field $F(t)=F_{0} \cos \omega t$. They developed a theory to model the multimode fields of a turbulent plasma and obtained reasonable agreement with their experimental results. We note the experimental conditions described in Eq. (4.7) and Table I of Ref. 35 corresponded for $n=13$ to $F_{o} / F_{\text {at }} \approx 0.03$ au and $\omega / \omega_{a t} \simeq 0.05$. These values are not too far below those needed for ionization processes to become important. Their theory ${ }^{35}$ 
ignored such processes. Sanchez and Bengston 37 observed Stark-broadening and satellites of lines in a turbulent helium plasma and found existing theory incapable of explaining their results quantitatively.

10. Fast-beam Studies of Hydrogen Spectra in Intense Microwaves.- Bayfield ${ }^{38}$ first proposed a fast-beam, laser experiment for production and detection of satellite levels of $\mathrm{H}$ (high $\mathrm{n}$ ) atoms in a single microwave field. The present author ${ }^{39}$ demonstrated the feasibility of this class of experiments with an important modification: The fast-beam was passed through two spatially-separated regions of microwave field whose strengths could be adjusted independently. $\mathrm{CO}_{2}$ laser photons were used to drive transitions from initial $n_{i}=10$ levels with an unmeasured distribution of substates to final high $n_{f}$ levels in the presence of the first microwave field, which produced the satellite structure being investigated. The second field was used to detect by microwave ionization those atoms that were pumped by both the laser and microwave photons in the first field. Thus, the satellite-producing field could be weaker than that needed to ionize the atoms. The frequency of the continuous $\mathrm{CO}_{2}$ laser was Doppler-tuned in the atomic rest frame by varying the beam velocity. Since transitions to $n_{f}>30$ levels were studied, we expect that if the effective dipole moment $d$ of the atoms in the first field scaled as $n^{2}$, the moment $d\left(n_{f}\right)$ of the final level was at least an order of magnitude larger than the moment $d\left(n_{j}=10\right)$ of the initial level. Therefore, the ratios of $\mathrm{dF}_{\mathrm{o}} / \hbar \omega$ that enter into the Bessel functions in Eq. (3) were much larger for the final level, and its satellites played a more important role than those of the initial level.

The results of that first unpublished experiment ${ }^{39}$ were included in a recent review ${ }^{2}$, from which Fig. 14 is taken. The hatched curves show experimental data for the production of the $\mathrm{P}_{f}=+1,+2$ satellites of $H\left(\mathrm{n}_{\mathrm{f}}=48\right)$ in a $9.91 \mathrm{GHz}$ field with peak amplitude $F_{0}(A)$. The abscissa of the figure is a quadratic $F_{0}(A)$ scale (proportional to the power dissipated in the microwave cavity) normalized to the field $F_{o}($ ion $) \simeq 90 \mathrm{~V} / \mathrm{cm}$ that was observed to ionize the $H\left(n_{f}=48\right)$ atoms. We see that the $p_{f}=1,+2$ signals peak at values of $F_{o}(A)$ less than $10 \%$ of $F_{o}$ (ion). The dashed curves show theoretical signal curves obtained using the form of the amplitude given by Eq. (3). The $p_{f}=+1$ dashed curve was normalized by adjusting the magnitude and argument (magnitude of $d_{f}$ ) of the first maximum of $J_{1}{ }^{2}$ to agree with the position and height of the peak of the experimental curve. The same normalization parameters were used with the function $\mathrm{J}_{2}^{2}$ to plot the dashed $\mathrm{p}_{\mathrm{f}_{2}}=+2$ curve. The fitted dipole moment is $d_{f}=2.6 \times 10^{3}$ au, or scaled down by $n^{2}, d_{f} / n_{f} \simeq 1$ for $\mathrm{n}_{\mathrm{f}}=48$. This is a rather reasonable result; the permanent dipole moment in the microwave field was about one scaled atomic unit $\left(\mathrm{au} / \mathrm{n}^{2}\right)$. According to Delone et a1. ${ }^{31}$, the linear interaction of the atoms with the oscillatory field dominates the quadratic interaction when $n^{4} F_{o} \ll 0.06$ au, or $F_{o} \ll 60 \mathrm{~V} / \mathrm{cm}$ for $n_{f}=48$, Since in Fig. $14 \mathrm{~F}_{\mathrm{o}}(\mathrm{A}) \leq 10 \mathrm{~V} / \mathrm{cm}$, the experiment fulfilled this condition.

Bayfield et al. 40 recently published the results of a more extensive study of the spectra of $\mathrm{H}\left(\mathrm{n}_{\mathrm{f}}=44-67\right)$ atoms in a $5.9-8.0 \mathrm{GHz}$ microwave field. They used a "two-field" technique similar to the one mentioned above. Fig. 15 shows two of their spectra for excitation of $n_{i}=10$ atoms to satellites of $n_{f}=43-45$. The higherpower spectrum in the lower frame exhibits more complicated, overlapping series of satellites (up to $\left|p_{f}\right|=15$ ) compared to the lower-power spectrum in the upper frame. Since the zero-field hydrogen levels ( $p_{f}=0$ satellites) are unequally spaced ( $n$ to $(n+\Delta n)$ spacing equal to $\Delta n / n^{3}$ au) and the satellites of a given $n$-level are equaliy spaced, the satellite patterns of different $n$-levels are out of step. In the limit of a huge number of satellites being appreciably populated in a strong field, one would expect the empty spaces between the zero-field levels to fill up with a kind of "lumpy continuum." The analogy 20 between the problems of multiphoton excitation and ionization of hydrogen atoms by a strong microwave field and of multiphoton dissociation of polyatomic molecules by an intense, infrared laser field should be mentioned here. The lumpy continuum in the present hydrogen problem is similar to the quasi-continuum of upper levels in the molecular problem (see Ref. 22 for a useful list of references). 


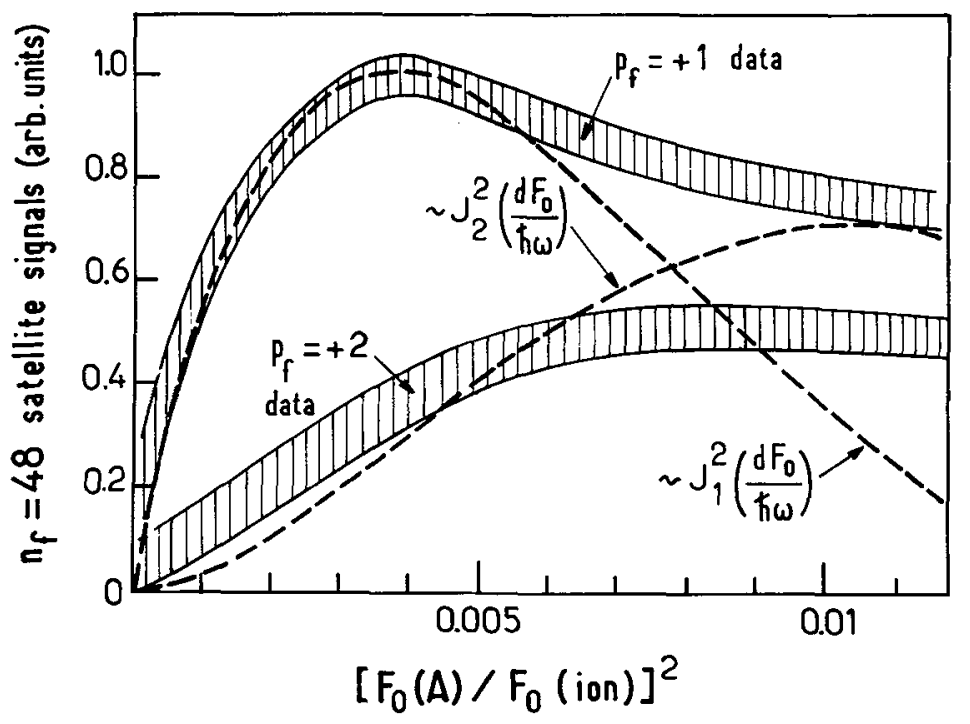

Fig. 15.- Cross-hatched areas: Experimental signals for $\mathrm{CO}_{2}$ laser-induced transitions from the $p_{i}=0$ satellite level of $H\left(n_{i}=10\right)$ to the $p_{f}=+1$, +2 satellite levels, respectively, of $\mathrm{H}\left(\mathrm{n}_{\mathrm{f}}=48\right)$ in a $9.91 \mathrm{GHz}$ electric field whose peak field strength was $F_{0}(A)$. $F_{0}(i \circ)=90 \nabla 7 \mathrm{~cm}$ was the field strength observed to ionize the $H\left(n_{f}=48\right)$ atoms. Dashed curves: Theoretical curves based on Eq. (3) that are proportional to the square of the Bessel functions of order $p_{f}$. The normalization procedure is discussed in the text.

Bayfield et al. 40 measured excitation curves similar to those shown in Fig. 14, and saw no oscillations past the first maximum that one would expect according to the extrema and zeroes of Bessel functions, Eq. (3). They ascribed the deviation between the "theoretical" and experimental curves at higher values of $F_{0}$ as due to the $\mathrm{n}_{\mathrm{f}}$-satellite levels having a spread in the value of the dipole moment $d_{f}$. This would cause a spread in the value of the argument of each Bessel function amplitude, Eq. (3), and for each different $d_{f}$-value, the squared Bessel function curve would peak at a different value of $F_{0}$. Some kind of average $d_{f}-v a l u e$ can adequately explain the low $-F_{0}$ part up to the first maximum of each curve, but the spread in $\mathrm{d}_{f}$-values changes the shape of each curve at higher $\mathrm{F}_{\mathrm{o}}$-values. For $\mathrm{n}_{\mathrm{f}}-\mathrm{levels}$ between 44 and 67 , and for frequencies $5.9-8.0 \mathrm{GHz}$, they found that the power at which each $p_{f}=-1$ satellite peaked was proportional to $\omega^{(3.0 \pm 0.5)} \mathrm{n}^{(-4.0 \pm 0.2)}$; the corresponding value of $d_{f}$ varies at $n^{2} \omega^{-\frac{1}{2}}$.

Stehle used a quantal forward scattering method to model theoretically the twofrequency (laser + microwaves) excitation of $H\left(n_{i}\right)$ atoms to $n_{f}-s t a t e s$. (The present author appreciates receipt of a pre-print on this work from Professor stehle before its publication.) His theory makes a prediction that could be tested in future experiments: The excitation amplitude for each satellite is still weighted by its pth-order Bessel function, Eq. (3), but all satellite-amplitudes share a common factor $\mathrm{J}_{\mathrm{o}}\left(2 \mathrm{dF}_{\mathrm{o}} / \mathrm{h} \omega\right)$. This new factor would make all satellites disappear together at values of $\mathrm{F}_{0}$ corresponding to the zeroes of $\mathrm{J}_{0}$. If the experimental situation includes a spread of $d_{f}$-values, these would have to be taken into account in applying the theory. We mention that modification and even vanishing of the Lande g-factor in atomic magnetic resonance experiments in a strong oscillating magnetic field $B_{1}$ was observed and explained theoretically using the "dressed-atom" formalism 


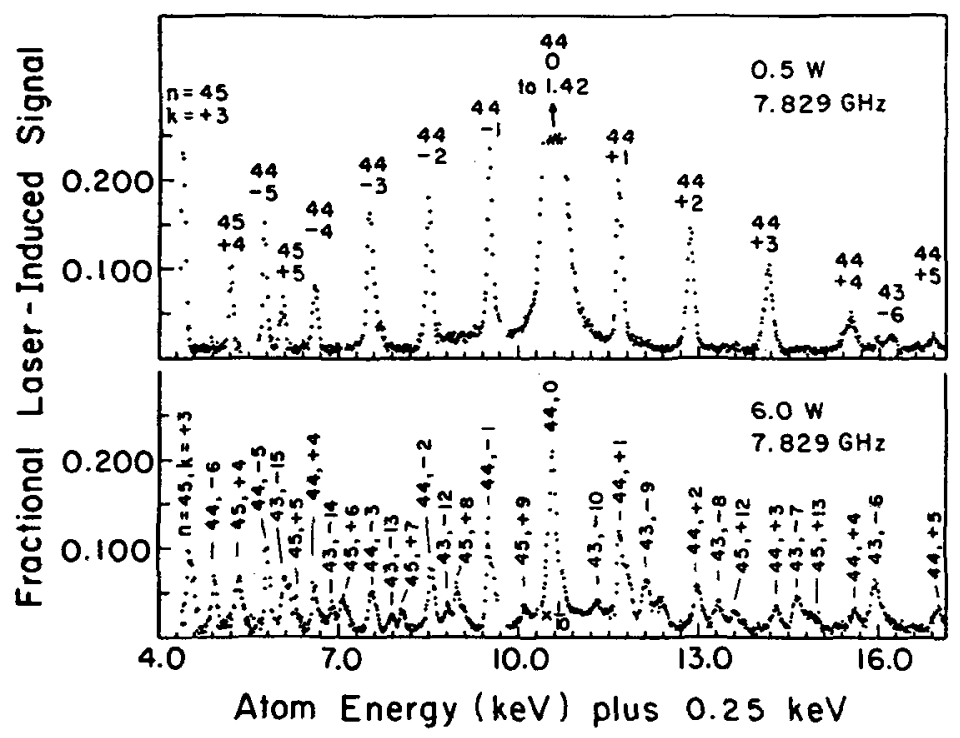

Fig. 16.- Excitation spectra (Ref. 40) in hydrogen atoms for $\mathrm{CO}_{2}$ laser-driven transitions from satelitite levels of the $n_{i}=10$ manifold to those of the $n_{f}=43,44$, or 45 manifolds. Assuming that only the $p_{i}^{i}=0$ satellites of the $n_{i}=10$ manifold contribute, the additional numbers near the peaks label the orders $p_{f}$ of the satelites populated in the $\mathrm{n}_{f}$-manifolds. The fixed-frequency $\mathrm{CO}_{2}$ laser radiation was Doppler-tuned in the atomic rest frame with variation of the kinetic energy of the atomic beam. Since lower energy atoms spent a longer time in the waveguide field region, the lower energy resonance peaks are more intense. The $p_{f}=0$ resonances contain large contributions from atoms pumped outside the waveguide field region. (taken from Ref. 40).

by Cohen-Tannoudji and Haroche many years ago (Ref. 32, Section II.B.) The effective g-factor (or, equivalently, the effective magnetic moment) in the intense field also displayed a zeroth-order Bessel function dependence on $\mathrm{B}_{1}$.

It is clear that future experiments will be much improved if one were to use $\mathrm{H}\left(\mathrm{n}_{i}=10\right)$ atoms prepared with a single value of $\mathrm{d}_{i}$, that is, in a single stark substate. These methods have been developed for static field studies $1,12,13$ and must now be applied to the microwave studies. It is not yet known, however, what happens to a hydrogen atom prepared in a single, static Stark state when it passes into a microwave electric field. If the microwave electric field is collinear with the static field direction, the $|\mathrm{m}|$-quantum number will certainly be conserved, but it is not clear that the value of the static dipole moment will be. Perhaps a cleaner situation will be to superimpose collinear static and microwave electric fields, but some of the same uncertainties about whether the value of the dipole moment remains constant will still have to be addressed.

11. Mixed-field Studies.- If the hydrogen atom were exposed to a microwave field plus additional static or oscillatory, magnetic or electric fields, the problem would obviously be made more complex. Such a situation is beyond the scope of the present paper, but a few experimental and theoretical papers have been published which point the way to future studies. We have already mentioned above the plasma physics experiments which investigated modifications of field-free atomic spectra by internal, plasma fields. Some of these fields can be described as quasi-static and others as dynamic. Gallagher and Levine ${ }^{4}$ reported observation of satellites of $\mathrm{H}_{B}-1$ ines in a turbulent hydrogen plasma that were ascribed to resonance effects between ortho- 
gonal components of dynamic and quasi-static internal electric fields. These resonance effects, which produce spectra dramatically different from the Stark spectrum of each field acting independently, were first investigated numerically by Cohn et a1.42 More recently, Gavrilenko and oks 43 have analytically confirmed and extended the theoretical work of Cohn et al. 42 They find that fields with fixed and random phases, respectively, exert different resonant action on the hydrogen spectral lines that start from levels with $n \geq 3$.

An important series of future experiments will use collision-free beams of hydrogen atoms laser-excited into individual (resonant-) satellite states produced by mixed, applied fields. This will be a cleaner experimental situation for studying the field-effects themselves than is provided by the internal fields in a turbulent plasma.

12. Additiona1 Fast-beam Experiments.- Our group at Yale (Mariani, van de Water, and Koch) recently joined with a group (Gemmel, Kanter, Schneider, et al.) at the Dynamitron Accelerator at Argonne National Laboratory to investigate further the influence of Rydberg atoms on measured energy spectra of "convoy electrons" produced by fast ions incident on a thin foil (for an extensive review of convoy electron studies, see Ref. 44). These electrons (called "cusp electrons" in ion-atom collisions in a gas target) emerge from the collision with nearly the same velocity and direction as the unscattered ions or forward-scattered ions that have captured target electron(s) into a bound state(s). For example, convoy electrons produced in $0.5 \mathrm{MeV} \mathrm{H}{ }^{+}$-foil interactions emerge with a kinetic energy about a factor ( $\mathrm{m}_{\mathrm{e}} / \mathrm{m}_{\mathrm{p}}$ ) smaller, or near $272 \mathrm{eV}$. The Argonne group showed previous $1 \mathrm{y}^{45}$ that electrons captured into bound Rydberg states could be ionized by electric fields in the electron spectrometer and elsewhere in the apparatus in such a way as to contribute to the apparent convoy-electron peak.

Since the analysis of the experiments is still in progress, we do not go into any detail here. We used a $9.91 \mathrm{GHz}$ microwave cavity to ionize Rydberg atoms after their point of production in a thin (2-5 micrograms $\left./ \mathrm{cm}^{2}\right)$ carbon foil and before their entrance into the electron spectrometer used to observe the convoy-electron spectra. Because of its high frequency, the microwave electric field negligibly affected the "true" convoy electrons. The foil could also be electrically biased to shift the energy of the convoy electrons relative to those produced by microwave ionization of the Rydberg atoms. We were able to resolve clearly these two sources of electrons in collisions of $0.5-2 \mathrm{MeV} \mathrm{H}{ }^{+}$and $\mathrm{He}^{+}$atomic ions and $\mathrm{H}_{2}^{+}$molecular ions with the foil. We are interested to see if an analysis of the data will give us preliminary experimental information on the continuum electron energy distribution produced in microwave ionization of hydrogen and helium Rydberg atoms.

13. Conclusions.- It is useful to summarize here the conclusions presented throughout the paper. The interactions of Rydberg atoms with an intense microwave electric field are not yet well understood. At the same field strength, multiphoton excitation and ionization can proceed at comparable rates, and each displays a different pattern of frequency-dependent resonances in hydrogen. To date, classical theory has furnished the only limited, quantitative agreement with hydrogen microwave ionization experiments. It also gives insight into some of the complicated motions of the atomic electron in the oscillatory field, but it ignores quantal tunneling processes that may be important in some cases. Tunneling has been shown to be important in static field ionization. There may be fundamental questions about a "stochastic mechanism" for ionization, but this has only been examined in a classical context which treats the hydrogen atom in the intense field as a nonlinear, classical dynamical system. If quantal effects are important, new questions are raised because the nature of the onset of stochasticity in quantum systems is poorly understood. Because of their very different behavior in an intense microwave field, non-hydrogenic Rydberg atoms should not be used to test hydrogen theory. Though we are able to explain at least some threshold features of the microwave ionization of helium Rydberg atoms, we are not yet able to explain most of the structure in the ionization curve. 
Quantum mechanics deals well with some aspects of atomic structure in the oscillatory field, but it has not yet been investigated carefully for fields strong enough to cause appreciable ionization. Recent theory predicts effects such as the disappearance of the satellite spectrum associated with a given n-level at certain values of the field strength $F$. This could be interpreted as the field-induced modification and vanishing of the atomic electric dipole moment at certain values of $F_{O}$, and, therefore, the electric resonance analog of the modification and vanishing of the Lande g-factor in magnetic resonance of atoms in a strong oscillating magnetic field.

Future microwave experiments will use available techniques for production of hydrogen atoms in individual Rydberg substates. These will allow more detailed studies of the atomic structure in the intense field as more refined excitation and ionization experiments.

14. Acknowledgments.- The author appreciates having shared many interesting discussions on a number of the subjects treated in this paper with his past and present Yale colleagues, particularly J.E. Bayfield, D.R. Mariani, and W. van de Water. He is grateful to the U.S. National Science Foundation for support of much of this work and to the A.P. Sloan Foundation for award of a Fellowship. He appreciates the work of the organizers of this Colloquium and the support given it by the French C.N.R.S.

\section{REFERENCES}

1. KOCH P.M., in ATOMIC PHYSICS, Vol. 7, D. Kleppner and F.M. Pipkin, eds. (Plenum, New York, 1981).

2. KOCH P.M., in RYDBERG STATES OF ATOMS AND MOLFCULES, R.F. Stebbings and F.B. Dunning, eds. (Cambridge University Press, New York, to be published 1982).

3. BAYFIELD J.E., in MULTIPHOTON PROCESSES, J.H. Eberly and P. Lambropoulos, eds. (Wiley, New York, 1978).

4. BAYFIELD J.E., Phys, Rep. 51 (1979) 317.

5. BAYFIELd J.E., Prog. Ouant. E1ec. 6 (1980) 219.

6. LEOPOLD J.G. and PERCIVAL I.C., Phys。Rev。 Lett. 41 (1978) 944.

7. LEOPOLD J.G. and PERCIVAL I.C., J. Phys. B12 (1979) 709.

8. JONES D.A., LEOPOLD J.G., and PERCIVAL I.C., J. Phys. B13 (1980) 31.

9. ZASLAVSKY G.M., Phys. Rep. $\underline{80}$ (1981) 157.

10. MEERSON B.I., OKS E.A., and SASOROV P.V., Pis'ma Zh. Eksp. Teor. Fiz. 29 (1979) 79 [Sov. Phys.-JETP Lett. 29 (1979) 72].

11. DELONE N.B., ZON B.A., and KRAINOV V.P., Zh. Eksp. Teor. Fiz. 75 (1978) 445 [Sov. Phys.-JETP 48 (1978) 223].

12. KOCH P.M., and MARIANI D.R., J. Phys, B13 (1980) L645.

13. KOCH P.M., and MARIANI D.R., Phys. Rev. Lett. 46 (1981) 1275.

14. DAMBURG, R.J. and KOLOSOV V.V., J. Phys. BI2 (1979) 2637.

15. BANKS D. and LEOPOLD J.G., J. Phys. B11 (1978) L5, 37. 
16. KELDYSH L.V., Zh. Eksp. Teor. Fiz. 47 (1964) 1945 [Sov. Phys,-JETP 20 (1965) $1307]$.

17. PERELOMOV A.M., POPOV V.S., and KUTNETSOV V.P., Zh. Eksp. Teor. Fiz. 54 (1968) 841 [Sov. Phys,-JETP 27 (1968) 451].

18. REISS H.R., Phys. Rev. A22 (1980) 1786.

19. BAYFIELD J.E. and KOCH P.M., Phys. Rev. Lett, 33 (1974) 258.

20. BAYFIELD J.E., GARDNER L.D., and KOCH P.M., Phys, Rev. Lett. 39 (1977) 76.

21. Gersten J. and MITTLEMAN M.H., Phys. Rev. All (1975) 1103.

22. ZeWAIL A.H., Phys. Today 33 (1980) 27.

23. JEYS T.H., FOLTZ G.W., SMITH K.A., BEITING E.J., KELLERT F.G., DUNNING F.B., and STEBBINGS R.F., Phys. Rev. Lett. 44 (1980) 390.

24. ZIMMERMAN M.L., LITTMAN M.G., KASH M.M., and KLEPPNER D。, Phys. Rev. A20 (1979) 2251.

25. RUBBMARK J.R., KASH M.M., LITTMAN M.G., and KLEPPNER D., Phys. Rev. A23 (1981) 3107.

26. BLOCHINZEW D., Phys. Z. Sowjetunion 4 (1933) 501.

27. AUTLER S.H., and TOWNES C.H., Phys. Rev. 100 (1955) 703.

28. BAKSHI P., KALMAN G., and COHN A., Phys. Rev, Lett. 31 (1975) 1576.

29. HICKS W.W., Lawrence Berkeley Laboratory Report LBL-2470 (Dec. 1973) unplublished.

30. HICKS W.W. HESS R.A., and COOPER W.S., Phys. Rev. A 5 (1972) 490.

31. DELONE N.B., ZON B.A., KRAJNOV V.P. and KHODOVOI V.A., Usp. Fiz Nauk 120 (1976) 3 [Sov. Phys.-Usp. 19 (1976) 711].

32. COHEN-TANNOUDJI C., and HAROCHE S., J. Physique 30 (1969) 153.

33. WEINGARTSHOFER A., HOLMES J.K, , CAUDLE G., ClARKE E.M., and KRUGER H., Phys. Rev. Lett. 39 (1977) 269.

34. BARANGER M., and MOZER B., Phys. Rev. 123 (1961) 25.

35. NEE T.-J.A., and GRIEM H.R., Phys. Rev. AI4 (1976) 1853.

36. PROSNITZ D., WILDIAN D.W., and GEORGE E.V., Phys. Rev. A13 (1976) 891.

37. SANCHEZ A., and BENGSTON R.D., Phys. Rev, Lett. $\underline{38}$ (1977) 1276.

38. BAYFIELD J.E., Abstracts of Invited Papers, European Study Conference on Multiphoton Processes, Seillac, France, April 1975 (unpublished).

39. Косн P.M., Post-deadline paper presented at the Int'1 Conf. on Multiphoton Processes, Rochester, NY, June 1977 (unpublished).

40. BAYFIELD J.E., GARNDER L.D., GULKOK Y.Z., and SHARMA S.D., Phys. Rev. A24 (1981) 138.

41. GALLAGHER C.C., and LEVINE M.A., Phys. Rev. Lett. 30 (1975) 897. 
42. COHN A., BAKSHI P., and KALMAN G., Phys. Rev. Lett. 29 (1972) 324 [Erratum: Phys. Rev. Lett. 31 (1973) 620].

43. GAVRILENKO V.P., and OKS E.A., Zh. Eksp. Teor. Fiz. 80 (1981) 2150 [Sov. Phys.JETP 53 (1981) 1122].

44. BREINIG M. ELSTON S.B., HULDT S., LILJEBY L., VANE C.R., BERRY S.D., GLASS G.A.. SCHAUER M., Sellin I.A., AITON G.D., DATZ S., OVERBURY S., LAUBERT R., and SUTER M., Phys. Rev. A25 (1982) 3015.

45. VAGER Z., ZABRANSKY B.J., SCHNEIDER D., KANTER E.P., ZHANG G.Y., and GEMMEL D.S., Phys. Rev. Lett. 48 (1982) 592. 